\title{
GRAPH MAPPINGS AND POINCARÉ DUALITY
}

\author{
Eric M. Friedlander* and H. Blaine Lawson, JR.*
}

Poincaré duality is an old and fundamental result dating from the very birth of Topology in [P]. In modern terminology, this result asserts for a compact, oriented $m$-manifold $M$ that the mapping

$$
\mathcal{D}: H^{k}(M, \mathbb{Z}) \rightarrow H_{m-k}(M, \mathbb{Z})=H_{m-k}(M)
$$

defined by the cap product with the fundamental class $[M]$ of $M$ is an isomorphism of finitely generated abelian groups.

This duality is manifest in many ways in geometry. Locally it corresponds to the duality of forms and currents in the theory of de Rham. It appears in Hodge theory via the *operator on harmonic forms. In Poincaré's original work it can be described via barycentric subdivision and dual cell complexes. It is interesting to note that each of these geometric formulations ignore torsion.

In recent years, we have investigated topological abelian groups of algebraic cycles on a complex projective variety $X$. We have introduced a companion, contravariant construction of algebraic cocycles. A somewhat unexpected development in this study is the appearance of a graphing construction

$$
\Gamma^{a l g}: \mathcal{M o r}\left(X, S P^{d}\left(\mathbb{P}^{s}\right)\right) \rightarrow Z_{n}\left(X \times \mathbb{P}^{s}\right), \quad n=\operatorname{dim}_{\mathbb{C}} X, \quad d>0
$$

where $S P^{d}\left(\mathbb{P}^{s}\right)$ denotes the $d$-fold symmetric power of the complex projective space $\mathbb{P}^{s}$. This construction determines a weak homotopy equivalence ("algebraic duality")

$$
\mathcal{D}^{\text {alg }}: Z^{s}(X) \rightarrow Z_{n-s}(X)
$$

between corresponding topological abelian groups of cocycles and cycles whenever $X$ is smooth (see [L2] for an overview of these matters and related results). The fact that this duality plays a role in understanding motivic cohomology (cf. [F-V]) suggests that our approach to duality might be applicable in other contexts as well.

In this paper, we establish that our algebro-geometric duality map $\mathcal{D}^{\text {alg }}$ relating algebraic cocycles to algebraic cycles on a projective algebraic variety can be extended using geometric measure theory to give a new formulation (whose justification requires one of the many existing proofs of Poincaré duality) of the integral Poincaré duality isomorphism for a compact, oriented manifold. We suggest that our topological graphing construction $\Gamma^{t o p}$ sending a multi-valued Lipschitz map to its graph offers a new geometric perspective on the fundamental map (0.1).

\footnotetext{
*Partially supported by the N.S.F. .
} 
In $\S 1$, we quickly review relevant concepts from geometric measure theory. We then show that the Dold-Thom isomorphism of algebraic topology and the Almgren isomorphism of geometric measure theory are compatible, each relating homotopy groups of a topological group of 0-cycles to homology. We then begin our investigation of $\operatorname{Map}_{\text {Lip }}\left(A, S P^{d}(B)\right)$ which serves as our gemetric measure-theoretic model of "cocyles on $A$ with values in $B$."

Proceeding by analogy with a construction for algebraic varieties given in $[\mathrm{F}-\mathrm{M}]$, we establish a set theoretic graphing construction for Lipschitz maps in Theorem 2.4. We proceed to then prove that this graphing construction induces Poincaré duality. Theorem 3.6 verifies that our graphing construction determines a well defined and continuous graphing function $\Gamma^{\text {top }}$ with values in integral cycles (in the sense of geometric measure theory).

Using the Dold-Thom theorem, we represent a cohomology class $\alpha \in H^{k}(A, \mathbb{Z})$ as a multi-valued map from $A$ to the sphere $S^{k}$. For single-valued maps $f_{\alpha}: M \rightarrow S^{k}$ from an oriented closed $d$-manifold $M$ to the $k$-sphere $S^{k}$, it is classical that the Poincaré dual of $\alpha$ is represented by the oriented, codimension- $k$ submanifold $f_{\alpha}^{-1}\left(y_{0}\right)$ where $y_{0}$ is a regular value of $f_{\alpha}$. Our multi-valued analogue of this observation, Theorem 5.3, can be viewed as asserting that the duality map (0.1) is given as the map on connected components of the composition

$$
\operatorname{Map}_{\text {Lip }}\left(M, S P^{\infty}\left(S^{k}\right)\right) \stackrel{\Gamma^{t o p}}{\longrightarrow} \mathcal{Z}_{m}\left(M \times S^{k}\right) \rightarrow \mathcal{Z}_{m-k}(M)
$$

where the second map is given by slicing integral cycles as discussed in $\S 4$.

Our main theorem, Theorem 5.4, asserts that the composition (0.2) is a weak homotopy equivalence whose associated isomorphism on homotopy groups $\pi_{j}$ can also be identified with the duality isomorphism $\mathcal{D}: H^{k-j}(M ; \mathbb{Z}) \stackrel{\simeq}{\longrightarrow} H_{m-k+j}(M)$. Heuristically, this is suggested by the observation that cap product is represented (for real cohomology and homology) by the map which sends a differential $k$-form $\omega$ to the $(m-k)$-current on $M$ given by $\delta \mapsto \int_{M} \delta \wedge \omega$. The insufficiency of this heuristic argument is that the cap product we must consider is that defined on simplicial integral cochains on $A$ and not on differential forms. One easy consequence of our main theorem is the fact that the algebro-geometric duality map $\mathcal{D}^{\text {alg }}$ of [F-L2] is fully compatible with Poincare duality.

We point out that in the case of manifolds P. Gajer has introduced an inverse construction to our graphing construction $[\mathrm{G}]$.

We express our gratitude to Christian Haesemeyer for numerous helpful discussions. During the many years this paper has been in preparation to complete, both authors have benefited from the hospitality of IHES.

\section{§1. Geometric-measure-theoretic spaCes of CyCles AND COCYCleS}

In this first section, we quickly review various concepts arising in geometric measure

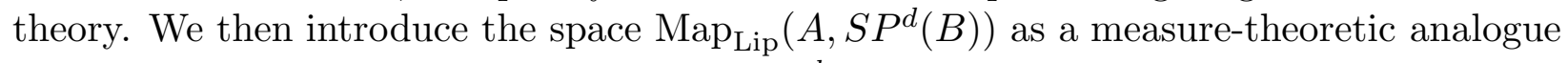
of the space of algebraic cocycles $\operatorname{Mor}\left(X, S P^{d}(Y)\right)$ first studied in [F-L1]. We recall that for any topological space $B$ and any positive integer $d, S P^{d}(B)=B^{\times d} / \Sigma_{d}$ denotes the $d$-fold symmetric power of $B$. These spaces play a fundamental role in the Dold-Thom formulation of homology $[\mathrm{D}-\mathrm{T}]$, and the spaces $\operatorname{Map}_{\mathrm{Lip}}\left(A, S P^{d}(B)\right)$ will play a similar role for cohomology. Our aim here is to express Poincaré duality in this context. 
Suppose now that $A$ is a compact polyhedron, i.e., a topological space admitting a finite triangulation by closed simplices. Let $A \subset \mathbb{R}^{N}$ be an embedding which is linear on the simplices (e.g., the obvious one when $N=$ the number of vertices). So embedded $A$ admits a tubular neighborhood $U \supset A$ with a Lipschitz retraction $\rho: U \rightarrow A$. Let $\mathcal{E}^{k}(U)$ denote the space of smooth differential $k$-forms on $U$ with the $C^{\infty}$-topology. The (pointwise) comass norm of a form $\omega \in \mathcal{E}^{k}(U),\|\omega\|$, is the supremum over the points $x$ in the support of $\omega$ of the value of $\omega$ applied to elements $\xi=\xi_{1} \wedge \cdots \wedge \xi_{k} \in \Lambda^{k}\left(T_{x}(U)\right)$ with $|\xi| \leq 1$.

The topological dual space of $\mathcal{E}^{k}(U)$ is the space of $k$-dimensional currents with compact support in $U$. For any such current $T$ there is defined a mass norm

$$
\operatorname{Mass}(T)=\sup \left\{|T(\omega)|: \omega \in \mathcal{E}^{k}(U) \text { with }\|\omega\| \leq 1\right\} .
$$

Important subsets of $k$-currents in $U$ are the Lipschitz polyhedral $k$-chains and the rectifiable $k$-currents, those which lie in the mass closure of the set of Lipschitz polyhedral $k$-chains. Important also are the integral $k$-currents (rectifiable $k$-currents with rectifiable boundaries), and integral $k$-cycles (integral $k$-currents with 0 boundary). The reader is referred to $[\mathrm{Fe}]$ for the relevant foundations. On these spaces one also has the flat norm defined by

$$
\|T\|_{b} \equiv \inf \{\operatorname{Mass}(S)+\operatorname{Mass}(R): T=R+\partial S \text { and } R, S \text { are rectifiable }\}
$$

We denote by

$$
\mathcal{R}_{k}(A) \supset \mathcal{I}_{k}(A) \supset \mathcal{Z}_{k}(A)
$$

the topological abelian groups of rectifiable $k$-currents, integral $k$-currents, and integral $k$-cycles respectively which are supported on $A$, provided with the flat norm topology. We shall also have occasion to consider the connected component of the identity $\mathcal{Z}_{k}(A)^{o}$ in the group $\mathcal{Z}_{k}(A)$.

Lemma 1.1. The groups $\mathcal{R}_{k}(A), \mathcal{I}_{k}(A)$ and $\mathcal{Z}_{k}(A)$ depend only on the PL-structure of $A$. In particular they are independent of the chosen embedding of $A$ into euclidean space.

Proof. This assertion follows straightforwardly from elementary geometric measure theory $[\mathrm{Fe}]$.

If $Z$ is a topological group, we shall use the notation $Z^{o}$ to denote the connected component of the identity of $Z$.

The following theorem of F. Almgren [A], extends to positive dimensional cycles the famous theorem of A. Dold and R. Thom [D-T].

Theorem 1.2. $[\mathrm{A}]$. Let $A \subset \mathbb{R}^{N}$ be a compact, local Lipschitz neighborhood retract. For any non-negative integer $j$ there is an isomorphism (natural with respect to Lipschitz maps)

$$
\mathcal{A}: \pi_{j} \mathcal{Z}_{k}(A) \stackrel{\simeq}{\longrightarrow} H_{k+j}(A) .
$$

Moreover, if $B \subset A$ is a closed subspace which is also a local Lipschitz neighborhood retract, then there is a natural isomorphism

$$
\mathcal{A}: \pi_{j}\left\{\mathcal{Z}_{k}(A, B) / \mathcal{I}_{k}(B)\right\} \stackrel{\simeq}{\longrightarrow} H_{k+j}(A, B)
$$


where $\mathcal{Z}_{k}(A, B)$ denotes the integral $k$-currents on $A$ whose boundary has support in $B$ and $\mathcal{I}_{k}(B)$ denotes the integral $k$-currents on $B$.

Finally, if $j>0$ or if $H_{k-1}(B) \rightarrow H_{k-1}(A)$ is injective, then the preceding isomorphism induces an isomorphism

$$
\mathcal{A}: \pi_{j}\left\{\mathcal{Z}_{k}(A) / \mathcal{Z}_{k}(B)\right\} \stackrel{\simeq}{\longrightarrow} H_{k+j}(A, B)
$$

Proof. The first two statements are precisely the main results of [A]. To obtain the third, observe that the evident embedding of topological groups

$$
\mathcal{Z}_{k}(A) / \mathcal{Z}_{k}(B) \subset \mathcal{Z}_{k}(A, B) / \mathcal{I}_{k}(B)
$$

has quotient equal to the discrete group $\operatorname{ker}\left\{H_{k-1}(B) \rightarrow H_{k-1}(A)\right\}$. Consequently, $\left(\mathcal{Z}_{k}(A, B) / \mathcal{I}_{k}(B)\right)^{o} \subset \mathcal{Z}_{k}(A) / \mathcal{Z}_{k}(B)$.

It is a "folk theorem" that the Dold-Thom and Almgren isomorphisms are compatible (cf. [Li2]). The following proposition establishes this compatibility.

Proposition 1.3. Let $X$ be a finite complex, let $\coprod_{d \geq 0} S P^{d}(X)$ denote the free abelian topological monoid on the points of $X$, and let $Z_{0}(X)$ be the naïve group completion of $\coprod_{d \geq 0} S P^{d}(X)$, the free abelian topological group on the points of $X$. Then the natural embedding $i_{X}: Z_{0}(X) \rightarrow \mathcal{Z}_{0}(X)$ induces an isomorphism on homotopy groups. Moreover, the composition

$$
\mathcal{A} \circ\left(i_{X}\right)_{*}: \pi_{*}\left(Z_{0}(X)\right) \rightarrow \pi_{*}\left(\mathcal{Z}_{0}(X)\right) \rightarrow H_{*}(X)
$$

equals the isomorphism of Dold and Thom [D-T].

Proof. The fact that $\left(i_{X}\right)_{*}$ is an isomorphism can be seen by observing that the topology of $Z_{0}(X)$ is the compactly generated topology associated to $\mathcal{Z}_{0}(X)$. Alternatively, this follows from the fact that $\mathcal{A} \circ\left(i_{X}\right)_{*}$ is the Dold-Thom isomorphism, which we now verify.

We see by inspection that $Z_{0}(-)$ converts disjoint unions of finite complexes to products of topological abelian groups. Almgren's theorem tells us that the canonical map $p_{A, B}$ : $\mathcal{Z}_{0}(X) \simeq \mathcal{Z}_{0}(A) \times \mathcal{Z}_{0}(B)$ associated to a decomposition $X=A \coprod B$ is a weak equivalence. Moreover, such a decomposition $X=A \coprod B$ identifies

$$
p_{A, B} \circ i_{X}: Z_{0}(A) \times Z_{0}(B)=Z_{0}(X) \rightarrow \mathcal{Z}_{0}(X) \rightarrow \mathcal{Z}_{0}(A) \times \mathcal{Z}_{0}(B)
$$

with the product $i_{A} \times i_{B}$. Consequently, to prove that $i_{X}$ is a weak equivalence, it suffices to consider the case in which $X$ is connected.

We recall that the Almgren isomorphism is natural in $X$ : given some some $f: S^{m} \rightarrow$ $\mathcal{Z}_{0}(X)$, one chooses a suitable polyhedral model of the $m$-cube with $m$-simplices $\sigma_{i}$ and a choice of map of chain complexes $\phi:\left(\mathcal{I}_{m}, \partial \mathcal{I}_{m}\right) \rightarrow\left(\mathcal{I}_{*}(X), 0\right)$ associated to $f$, and one sends $f$ to the homology class of $\phi_{*}\left(\sum_{i} \sigma_{i}\right)$. Almgren characterizes $\phi$ (depending upon $f$ ) up to chain homotopy by certain properties. The point is that this construction is natural in $X$ (although $\mathcal{I}_{m}$ requires subdivision, so for a different $X$ one might need to replace $\mathcal{I}_{m}$ by a complex involving a finer subdivision). 
Consider the natural diagram

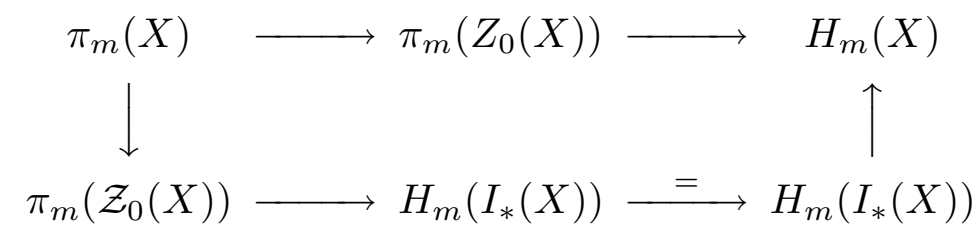

whose top row is the Hurewicz homomorphism in terms of the Dold-Thom isomorphism (the upper right arrow), whose left vertical map is induced by the continuous map $X \rightarrow$ $\mathcal{Z}_{0}(X)$, whose right vertical map is the natural identification, and whose bottom row is the Almgren isomorphism.

Essentially by inspection, this diagram is a commutative diagram of isomorphisms in the special case that $X=S^{m}$. By naturality, this tells us that the diagram commutes for all $X$. Consequently, $\mathcal{A} \circ\left(i_{X}\right)_{*}$ coincides with the Dold-Thom isomorphism on the image of the Hurewicz homomorphism.

Heuristically, we apply this argument with $X$ replaced by $Z_{0}(X)$. Since the latter is infinite dimensional, we apply it instead to the symmetric powers $S P^{d}(X)$ for each $d>0$, thereby concluding the commutativity of the square

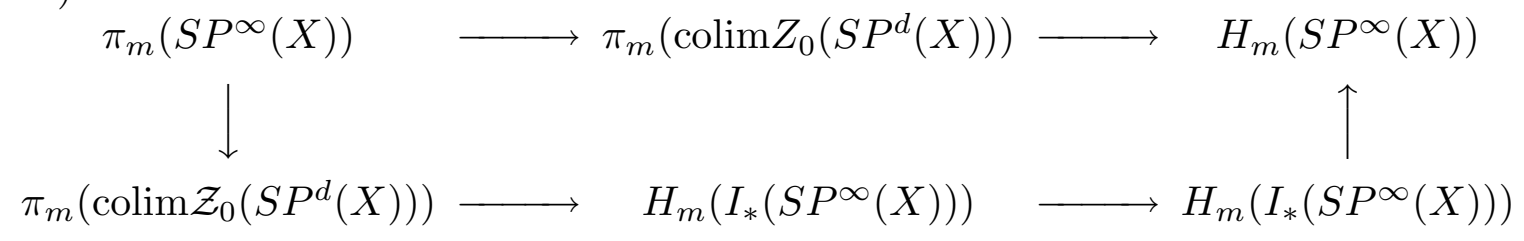

Finally, we apply the canonical retraction $H_{*}\left(S P^{\infty}(X)\right) \rightarrow H_{*}(X)$, characterized by the fact that it restricts to the identity on the image of $H_{*}(X) \rightarrow H_{*}\left(S P^{\infty}(X)\right)$ and is induced by a homomorphism of simplicial abelian groups (e.g., $Z(\operatorname{Sing.}(Z(X)) \rightarrow \operatorname{Sing} .(Z(X)))$. We conclude by observing that composition of this retraction with the top row of (1.3.1) equals the Dold-Thom map, whereas composition of the retraction with the bottom row and right vertical map of (1.3.1) equals $\mathcal{A}$.

We propose that the geometric measure-theoretic analogue of an algebraic cocycle on $X$ with values in $Y$, introduced in [F-L1], is a Lipschitz map from a compact polyhedron $A$ to some symmetric power $S P^{d}(B)$ of a compact polyhedron $B$. We denote by $\operatorname{Map}_{\text {Lip }}\left(A, S P^{d}(B)\right)$ the set of such maps provided with the topology of uniform convergence with bounded Lipschitz constant. In other words, the sequence $\left\{f_{n}\right\} \subset \operatorname{Map}_{\operatorname{Lip}}\left(A, S P^{d}(B)\right)$ converges to $f$ if and only if

$$
\left\|f_{n}-f\right\|_{\infty} \mapsto 0 \quad \& \quad \exists K \text { such that } \operatorname{Lipf}_{n} \leq K \text { for all } n
$$

Proposition 1.4. Let $A \subset \mathbb{R}^{N}$ be a compact Lipschitz neighborhood retract and $B$ a connected compact polyhedron. Then for any $d>0$ the continuous inclusion of mapping spaces

$$
\operatorname{Map}_{\mathrm{Lip}}\left(A, S P^{d}(B)\right) \rightarrow \operatorname{Map}_{\mathrm{cont}}\left(A, S P^{d}(B)\right)
$$


is a weak homotopy equivalence.

Proof. Choose open neighborhoods $A \subset U$ of $A \subset \mathbb{R}^{N}$ and $S P^{d}(B) \subset V$ of $S P^{d}(B) \subset \mathbb{R}^{M}$ with Lipschitz neighborhood retractions $\rho_{A}: U \rightarrow A, \rho_{B}: V \rightarrow S P^{d}(B)$. Let $A \subset U_{1} \subset U$ be a compact neighborhood of $A$. Given any continuous map $f: A \rightarrow S P^{d}(B)$, we can approximate $f \circ \rho_{A}: U \rightarrow A \rightarrow S P^{d}(B) \subset V$ by a smooth map $\tilde{f}: U \rightarrow V$ whose restriction $\left.\tilde{f}\right|_{U_{1}}$ can be made arbitrarily close to $\left.\left(f \circ \rho_{A}\right)\right|_{U_{1}}$; in particular, $\left.\tilde{f}\right|_{U_{1}}$ is homotopic to $f \circ \rho_{A}$. (This is achieved by convolution of $f \circ \rho_{A}$ with an approximate identity of $U_{1}$; cf. [Fe, 4.1.2].) Then

$$
\left.\rho_{B} \circ \tilde{f}\right|_{A}: A \subset U \rightarrow V \rightarrow S P^{d}(B)
$$

is a Lipschitz map homotopic to $f$. Thus, (1.4.1) induces a surjection on connected components.

If $f: A \rightarrow S P^{d}(B)$ is itself a Lipschitz map, then so is $f \circ \rho_{A}$ and the homotopy $F: A \times I \rightarrow B$ relating $f$ and $\left.\rho_{B} \circ \tilde{f}\right|_{A}$ is also Lipschitz (assuming that $\tilde{f}$ is sufficiently close to $\left.f \circ \rho_{A}\right)$. Hence, if two Lipschitz maps $f, g: A \rightarrow S P^{d}(B)$ are homotopic, the preceding process applied to this homotopy determines a Lipschitz homotopy relating $f, g$ and thus a path from $f$ to $g$ in $\operatorname{Map}_{\text {Lip }}\left(A, S P^{d}(B)\right)$. In other words, (1.4.1) also induces an injection on connected components.

Fix some Lipschitz map $f: A \rightarrow S P^{d}(B)$ and consider the restriction of (1.4.1) to connected components containing $f$ :

$$
\operatorname{Map}_{\text {Lip }}\left(A, S P^{d}(B)\right)_{f} \subset \operatorname{Map}_{\text {cont }}\left(A, S P^{d}(B)\right)_{f}
$$

For some $j>0$, we consider a pointed map $F: S^{j} \rightarrow \operatorname{Map}_{\text {cont }}\left(A, S P^{d}(B)\right)_{f}$. Let $G$ : $S^{j} \times A \rightarrow S P^{d}(B)$ denote the adjoint of $F$; in particular, the restriction of $G$ to $\{\infty\} \times A$ equals $f$. Arguing as above with $A$ replaced by $A^{\prime}=S^{j} \times A$, we obtain a Euclidean neighborhood $A^{\prime} \subset U^{\prime}$ with Lipschitz deformation retraction $\rho_{A^{\prime}}: U^{\prime} \rightarrow A^{\prime}$ together with a compact neighborhood $A^{\prime} \subset U_{1}^{\prime} \subset U^{\prime}$. Once again, we may find smooth maps $\tilde{G}: U^{\prime} \rightarrow V$ whose restrictions to $U_{1}^{\prime}$ can be made arbitrarily close to the restriction of $G \circ \rho_{A^{\prime}}: U^{\prime} \rightarrow A \times S^{j} \rightarrow S P^{d}(B) \subset V$. Thus, $\left.\rho_{B} \circ \tilde{G}\right|_{S^{j} \times A}: S^{j} \times A \rightarrow S P^{d}(B)$ is a Lipschitz map homotopic to $G$. Moreover, as argued above, the homotopy relating $\left.\rho_{B} \circ \tilde{G}\right|_{S^{j} \times A}$ and $G$ can be taken to be Lipschitz when restricted to $\{\infty\} \times A \times I$. Thus, we can modify the homotopy to yield a homotopy $H: S^{j} \times A \times I \rightarrow S P^{d}(B)$ relating $G$ to a Lipschitz map $G^{\prime}$ with the property that each restriction $\left.H\right|_{\infty \times A \times\{t\}}$ equals $f$. So constructed, $G^{\prime}$ corresponds to $F^{\prime}: S^{j} \rightarrow \operatorname{Map}_{\text {Lip }}\left(A, S P^{d}(B)\right)$ homotopic via a base-point preserving homotopy to $F$. We have therefore proved that $(1.4 .1)_{f}$ induces a surjection on homotopy groups.

The proof that $(1.4 .1)_{f}$ induces an injection on homotopy groups is obtained by modifying the proof of surjectivity given immediately above as in the proof of injectivity on connected components.

Corollary 1.5. Let $A \subset \mathbb{R}^{N}$ be a compact Lipschitz neighborhood retract and $B$ a connected compact polyhedron with chosen non-degenerate base point. Then the inclusion of mapping spaces with the compact-open topology

$$
\operatorname{colim}_{d} \operatorname{Map}_{\text {Lip }}\left(A, S P^{d}(B)\right) \subset \operatorname{Map}_{\text {cont }}\left(A, \mathcal{Z}_{0}(B)^{o}\right)
$$


is a weak homotopy equivalence.

Proof. Because (directed) colimits commute with taking homotopy groups, we conclude that the colimit with respect to $d$ of $(1.4 .1)$,

$$
\operatorname{colim}_{d} \operatorname{Map}_{\mathrm{Lip}}\left(A, S P^{d}(B)\right) \rightarrow \operatorname{colim}_{d} \operatorname{Map}_{\mathrm{cont}}\left(A, S P^{d}(B)\right),
$$

is a weak equivalence. On the other hand, the connectivity of $S P^{d}(B) \rightarrow S P^{\infty}(B)$ increases as $d$ increases [Mi], and by [D-T] the map $S P^{\infty}(B) \rightarrow Z_{0}(B)^{\circ}$ is a weak equivalence. Since any map from a compact space $A^{\prime}$ to $S P^{\infty}(B)$ has image in some $S P^{d}(B)$, we conclude that the composition

$$
\operatorname{colim}_{d} \operatorname{Map}_{\text {cont }}\left(A, S P^{d}(B)\right) \rightarrow \operatorname{Map}_{\text {cont }}\left(A, S P^{\infty}(B)\right) \rightarrow \operatorname{Map}_{\text {cont }}\left(A, Z_{0}(B)^{o}\right)
$$

is a weak equivalence. Thus, the corollary follows by applying the observation that the topology on $Z_{0}(X)$ is the compactly generated topology associated to $\mathcal{Z}_{0}(X)$.

\section{$\S 2$. Construction of Graphs}

In this section, we present our construction of the graph $\Gamma(f)$ of a Lipschitz map $f$ : $A \rightarrow B$ between compact polyhedra.

In order to ultimately apply our duality results in algebraic geometry, we want to consider objects somewhat more general than manifolds. The following is our working definition of a compact oriented pseudo-manifold. This class of objects includes compact oriented manifolds and complex projective varieties.

Definition 2.1. Let $A$ be a compact connected polyhedron. $A$ is said to be a compact pseudo-manifold of dimension $m$ if $A$ admits a triangulation $\mathcal{T}$ satisfying:

(1) Every simplex of $\mathcal{T}$ is contained in the closure of some $m$-simplex $\tau \in \mathcal{T}(m)$.

(2) For some smooth closed manifold $M$ equipped with a smooth triangulation, there exists a polyhedral map $p: M \rightarrow A$ restricting to a homeomorphism $M-M^{\prime} \rightarrow$ $A-s k_{m-2} A$, where $M^{\prime} \subset M$ is a subcomplex of dimension $\leq m-2$.

If $A$ is a compact pseudo-manifold provided with a fundamental class $[A] \in H_{m}(A, \mathbb{Z})$ such that $p: M \rightarrow A$ can be chosen as above with $M$ oriented and $p_{*}([M])=[A]$, then we say that $A$ is a compact oriented pseudo-manifold

Note. We will assume that $A$ has been provided with a piecewise smooth Riemannian metric compatible with the triangulation $\mathcal{T}$ (e.g., a piecewise flat metric), so that one may discuss Lipschitz functions on $A$. This class of Lipschitz functions is independent of the particular choice of metric.

The following important theorem, which we shall often apply without explicit reference, provides a method of constructing currents on open subsets of Euclidean space with given support. 
Theorem 2.2. (Kirszbraun's theorem; cf. [Fe;2.10.43]) Let $S \subset \mathbb{R}^{m}$ be an arbitrary subset of $\mathbb{R}^{m}$ and consider $f: S \rightarrow \mathbb{R}^{n}$, a Lipschitz map with Lipschitz constant $K$. Then there exists an extension $\tilde{f}: \mathbb{R}^{m} \rightarrow \mathbb{R}^{n}$ which is also a Lipschitz map with Lipschitz constant $K$.

We shall call such an $\tilde{f}$ as in Theorem 2.2 a Kirszbraun extension of $f$.

Using the Kirszbraun's theorem, we define push-forwards of basic currents via Lipschitz maps.

Lemma 2.3. Let $E \subset \mathbb{R}^{m}$ be a Borel set with compact closure, and let $\chi_{E}$ denote the characteristic function of $E$. Then the current $[E]=\chi_{E}\left[\mathbb{R}^{m}\right]$ (where $\mathbb{R}^{m}$ is given its standard orientation) defined by

$$
[E](\omega)=\int_{\mathbb{R}^{m}} \chi_{E} \omega
$$

for smooth $m$-forms $\omega$ is a rectifiable $m$-current in $\mathbb{R}^{m}$. Furthermore, if $f: E \rightarrow \mathbb{R}^{n}$ is a Lipschitz mapping, then the rectifiable $m$-current $f_{*}[E] \stackrel{\text { def }}{=} \widetilde{f}_{*}[E]$ is independent of the choice of the Kirszbraun extension $\tilde{f}: \mathbb{R}^{m} \rightarrow \mathbb{R}^{n}$ of $f$.

Proof. We see that $[E]$ is rectifiable as follows. There exists a family of bounded open sets $U_{k}$ containing $E$ with $\mu\left(U_{k}-E\right)<1 / k$ where $\mu$ denotes Lebesgue measure. Choose a triangulation of $U_{k}$ and a finite union of $m$-simplices $P_{k} \subset U_{k}$ with $\mu\left(U_{k}-P_{k}\right)<1 / k$. Then $\mu\left(P_{k} \Delta E\right)<2 / k$ where $P_{k} \Delta E=\left(P_{k}-E\right) \cup\left(E-P_{k}\right)$. Since Mass $\left([E]-\left[P_{k}\right]\right) \leq \mu\left(P_{k} \Delta E\right)$ we see that $[E]$ lies in the mass closure of the Lipschitz polyhedral $m$-chains and is therefore rectifiable by definition.

The independence of the current $f_{*}[E]$ from the choice of Lipschitz extension $\tilde{f}$ is proved in $[\mathrm{Fe}, 4.1 .15]$.

The following theorem provides the construction of our topological graphing. We leave to the next section the verification that this graphing construction (at least upon restriction to a dense open subset of $\left.\operatorname{Map}_{\text {Lip }}\left(A, S P^{d}(B)\right)\right)$ takes values in integral cycles and is continuous.

Theorem 2.4. Let $A$ be a compact oriented pseudo-manifold of dimension $m$, let $B$ be a compact polyhedron, and let $d>0$ be a positive integer. Then there is a well defined set-theoretic function

$$
\Gamma: \operatorname{Map}_{\text {Lip }}\left(A, S P^{d}(B)\right) \rightarrow \mathcal{R}_{m}(A \times B)
$$

sending a Lipschitz map $f: A \rightarrow S P^{d}(B)$ to its "graph" $\Gamma(f)$ as a rectifiable current on $A \times B$.

Proof. Choose a triangulation $\mathcal{T}$ of $S P^{d}(B)$, compatible with the singular stratification, and lift $\mathcal{T}$ via the projection $\pi: B^{\times d} \rightarrow S P^{d}(B)$ to an invariant triangulation of $B^{\times d}$. Given $f \in \operatorname{Map}_{\text {Lip }}\left(A, S P^{d}(B)\right)$, consider a $k$-simplex $\Delta$ of $S P^{d}(B)$. Let $\widetilde{\Delta}$ be a $k$-simplex of $B^{\times d}$ with $\pi(\widetilde{\Delta})=\Delta$. Then lifting and projecting

$$
\begin{array}{r}
\tilde{\Delta} \subset B^{\times d} \stackrel{p_{i}}{\longrightarrow} B \\
\tilde{f} \nearrow \downarrow \\
f^{-1}(\Delta) \underset{f}{\longrightarrow} \Delta
\end{array}
$$


gives Lipschitz maps $f_{i, \Delta} \equiv p_{i} \circ \tilde{f}$ on $f^{-1}(\Delta)$. Up to reordering these maps are independent of the choice of $\widetilde{\Delta}$ above $\Delta$. If $\Delta$ is a face of $\Delta^{\prime}$, then the functions $\left\{f_{j, \Delta^{\prime}}\right\}$ restrict to give the functions $\left\{f_{j, \Delta}\right\}$ on $f^{-1}(\Delta) \subset f^{-1}\left(\Delta^{\prime}\right)$.

We now decompose $A$ into a finite disjoint union of Borel sets

$$
A=\coprod_{\Delta \in \mathcal{T}} A_{\Delta}
$$

where $A_{\Delta}=f^{-1}\left(\Delta^{o}\right), \Delta^{o}=\Delta-\partial \Delta$ and where $\Delta$ ranges over all the simplices of $\mathcal{T}$. (This gives a decomposition of the current $[A]=\sum_{\Delta}\left[A_{\Delta}\right]$.) For each $\Delta \in \mathcal{T}$ we define the rectifiable $m$-current $\Gamma_{\Delta}$ on $A \times B$ by

$$
\Gamma_{\Delta} \stackrel{\text { def }}{=} \sum_{i=1}^{d} \operatorname{graph}\left(f_{i, \Delta}\right) \stackrel{\text { def }}{=} \sum_{i=1}^{d}\left(F_{i, \Delta}\right)_{*}\left[A_{\Delta}\right]
$$

where $F_{i, \Delta}: A_{\Delta} \rightarrow A \times B$ is given by

$$
F_{i, \Delta}(x)=\left(x, f_{i, \Delta}(x)\right)
$$

Observe that $\left(F_{i, \Delta}\right)_{*}\left[A_{\Delta}\right]$ is a rectifiable current on $A \times B$ by Lemma 2.3 , and therefore the sum:

$$
\Gamma(f) \stackrel{\text { def }}{=} \sum_{\Delta \in \mathcal{T}} \Gamma_{\Delta}
$$

is a well-defined $m$-dimensional rectifiable current. Furthermore, by [Fe, 4.1.14] we see that for each $\Delta$

$$
\operatorname{Mass}\left(\left(F_{i, \Delta}\right)_{*}\left[A_{\Delta}\right]\right) \leq\left(\operatorname{LipF}_{i, \Delta}\right)^{m} \mu\left(A_{\Delta}\right) \leq d(1+\operatorname{Lipf})^{m} \mu\left(A_{\Delta}\right)
$$

where $\mu$ denotes Hausdorff measure in dimension $m$. Consequently we have

$$
\operatorname{Mass}(\Gamma(f)) \leq d(1+\text { Lipf })^{m} \operatorname{vol}(A)
$$

Remark 2.5. In the special case of simplicial maps, the graphing construction can be done much more transparently. Suppose $f: A \rightarrow S P^{d}(B)$ is a simplicial map with respect to triangulations of $A$ and $B$ as above. Let $\mathcal{T}^{j}(A)$ denote the set of oriented $j$-simplices of $A$. For each $m$-simplex $D \in \mathcal{T}^{m}(A)$ there is a simplex $\Delta$ in $B$ with $f(D) \subset \Delta$, and we can construct maps $f_{i}: D \rightarrow B, 1 \leq i \leq d$, by lifting and projecting as we did above. Let $\Gamma_{D}$ denote the sum of the graphs of these mappings. Then

$$
\Gamma(f)=\sum_{D \in \mathcal{T}^{m}(A)} \Gamma_{D}
$$


In this case one sees that

$$
\partial \Gamma(f)=\left.\sum_{D \in \mathcal{T}^{m}(A)} \Gamma_{D}\right|_{\partial D}=0
$$

where cancellation occurs on the codimension-one faces as usual because $A$ is an oriented pseudo-manifold.

For a general Lipschitz map $f: A \rightarrow S P^{d}(B)$, the graph cannot be re-expressed in these simplicial terms and the computation of $\partial \Gamma(f)$ is more complicated.

$$
\text { §3. } \Gamma^{\text {top }}: \operatorname{Map}_{\text {Lip }}\left(A, S P^{d}(B)\right) \rightarrow \mathcal{Z}_{m}(A \times B)
$$

Throughout this section $A$ and $B$ shall denote compact pseudo-manifolds of dimensions $m$ and $n$ respectively with $A$ oriented. We let $A_{0} \subset A$ be a closed subset, and $A^{\prime} \subset A$ the open complement of $A_{0}$. Beginning with the set function $\Gamma: \operatorname{Map}_{\mathrm{Lip}}\left(A^{\prime}, S P^{d}(B)\right) \rightarrow$ $\mathcal{R}_{m}(A \times B)$ of Theorem 2.4, we construct a continuous map $\Gamma^{t o p}: \operatorname{Map}_{\mathrm{Lip}}\left(A^{\prime}, S P^{d}(B)\right) \rightarrow$ $\mathcal{Z}_{m}\left(A \times B, A_{0} \times B\right)$ leading to a continuous group homomorphism $\operatorname{Map}_{\text {Lip }}\left(A^{\prime}, \mathcal{Z}_{0}(B)\right) \rightarrow$ $\mathcal{Z}_{m}\left(A \times B, A_{0} \times B\right)$. Our program is to show that $\Gamma$ is continuous and takes values in integral cycles when restricted to the dense subset of "good" maps in $\operatorname{Map}_{\text {Lip }}\left(A, S P^{d}(B)\right)$.

We first make explicit some of the geometric terminology we use in our analysis of the graphing construction.

The singular set $\Sigma \subset S P^{d}(B)$ consists of those $d$-tuples of points of $B$ with at least one repetition. A map $\tilde{f}: \mathbb{R}^{m} \rightarrow \mathbb{R}^{n}$ which is $C^{1}$ in a neighborhood of some $x \in \mathbb{R}^{m}$ is said to be transverse to an open $k$-simplex $\Delta$ of $\mathbb{R}^{n}$ if $d \tilde{f}^{*-1}\left(T_{\Delta, \tilde{f}(y)}\right) \subset T_{\mathbb{R}^{m}, x}$ has codimension $n-k$.

By choosing a $P L$ tubular neighborhood of $S P^{d}(B)$ for some $P L$-embedding $S P^{d}(B) \subset$ $\mathbb{R}^{N}$, we easily verify the following lemma.

Lemma 3.1. Choose a triangulation of $S P^{d}(B)$ such that the singular set $\Sigma$ is a subcomplex and let $S P^{d}(B) \subset \mathbb{R}^{N}$ be some PL-embedding. Then there exist a compact neighborhood $U$ of $S P^{d}(B)$ in $\mathbb{R}^{N}$, a Lipschitz retraction

$$
\pi: U \rightarrow S P^{d}(B)
$$

and a finite triangulation of $U$ with $S P^{d}(B)$ as a subcomplex such that $\pi^{-1}(\Sigma)$ is a subcomplex of codimension $\geq 1$.

We introduce a condition on Lipschitz maps $f: A \rightarrow S P^{d}(B)$ which insures that $\Gamma(f)$ is $\partial$-closed.

Definition 3.2. Suppose $A$ is a compact pseudo-manifold with a given triangulation $\mathcal{T}$ as in Definition 2.1. A Lipschitz map $f: A \rightarrow S P^{d}(B)$ is called good if $f$ is of the form $f=\pi \circ \tilde{f}$ for some Lipschitz retraction $\pi: U \rightarrow S P^{d}(B)$ of a compact neighborhood $U$ of $S P^{d}(B)$ satisfying the conditions of Lemma 3.1 and some $\tilde{f}: A \rightarrow U$ whose restriction to every simplex in $A$ is smooth and transverse to every open simplex of $\pi^{-1}(\Sigma) \subset U$.

We now proceed to analyze the construction $\Gamma$ of Theorem 2.2 when applied to good Lipschitz maps $f$. 
Proposition 3.3. Let $f: A \rightarrow S P^{d}(B)$ be a good Lipschitz map. Then the graph $\Gamma(f) \in R_{m}(A \times B)$ (as constructed in Theorem 2.4) is $\partial$-closed.

Proof. We first assume that $A$ is smooth, so that $M=A$. Recall that $S P^{d}(B)-\Sigma$ is the configuration space of sets of $d$ mutually distinct points in $B$. Let $G \subset\left(S P^{d}(B)-\Sigma\right) \times B$ be the tautological graph over $S P^{d}(B)-\Sigma$. The projection pr: $S P^{d}(B) \times B \rightarrow S P^{d}(B)$ restricts to a covering map $G \stackrel{\mathrm{pr}}{\longrightarrow} S P^{d}(B)-\Sigma$. Consider the fibre product

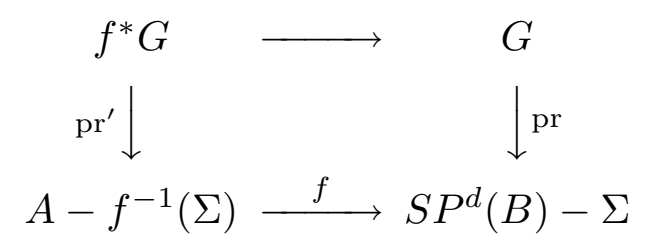

Then $\mathrm{pr}^{\prime}$ is also a covering map.

Observe that the closed subset $f^{*} G \subset\left(A-f^{-1}(\Sigma)\right) \times B$ determines an $m$-current $\Gamma(f)^{o}=\left[f^{*} G\right]$ on $\left(A-f^{-1}(\Sigma)\right) \times B$ which is given locally as the graph of a Lipschitz function over the base $A-f^{-1}(\Sigma)$. Since the fundamental class $[A]$ is a $\partial$-closed $m$-current on $A,\left[A-f^{-1}(\Sigma)\right]$ is a $\partial$-closed $m$-current on $A-f^{-1}(\Sigma)$ and thus $\Gamma(f)^{o}$ s a $\partial$-closed $m$-current on $\left(A-f^{-1}(\Sigma)\right) \times B$. By inspection, we see that the restriction of $\Gamma(f)$ to $\left(A-f^{-1}(\Sigma)\right) \times B$ equals $\Gamma(f)^{o}$. Thus we have proved that

$$
\operatorname{supp}\{\partial \Gamma(f)\} \subset f^{-1}(\Sigma) \times B .
$$

Since $f$ is good we have a lifting $\tilde{f}: A \rightarrow U$ which is transverse to $\pi^{-1}(\Sigma)$. This implies that the pre-image $\tilde{f}^{-1}\left(\pi^{-1}(\Delta)\right)$ for any simplex $\Delta$ of $\Sigma$ is a compact submanifold with corners of codimension $\geq 1$ in $A$, and thus so is $f^{-1}(\Sigma)$. Let $R$ be the set of regular codimension-1 manifold points of $f^{-1}(\Sigma)$. We will show that $\partial \Gamma(f)=0$ across $(R \cap A) \times B$.

Fix $x \in R$ and choose coordinates $\xi=\left(\xi_{1}, \ldots, \xi_{m}\right),\|\xi\|<1$, on a neighborhood $D$ of $x$ such that $\xi(x)=0$ and $\xi(R)$ is the hyperplane $\xi_{m}=0$. Now the map $f$ has $d$ distinct branches

$$
\begin{array}{lll}
\left\{f_{1}^{+}, \ldots, f_{d}^{+}\right\} & \text {over the set } & D^{+}=\left\{\xi:\|\xi\|<1 \text { and } \xi_{m}>0\right\} \\
\left\{f_{1}^{-}, \ldots, f_{d}^{-}\right\} & \text {over the set } & D^{-}=\left\{\xi:\|\xi\|<1 \text { and } \xi_{m}<0\right\}
\end{array}
$$

Each of the functions $f_{j}^{+}$has a Lipschitz extension to the closed half ball $\overline{D^{+}}=\{\xi$ : $\|\xi\|<1$ and $\left.\xi_{n} \geq 0\right\}$, and similarly each $f_{j}^{-}$has a Lipschitz extension to $\overline{D^{-}}$. To see this, note first that since $f$ is Lipschitz on all of $A$, each branch of $f$ over any subset of $A$ is also Lipschitz with the same Lipschitz constant. Hence the functions

$$
f_{j, \epsilon}^{+}\left(\xi^{\prime}\right) \stackrel{\text { def }}{=} f_{j}^{+}\left(\xi^{\prime}, \epsilon\right) \quad \text { where } \quad \xi^{\prime}=\left(\xi_{1}, \ldots, \xi_{n-1}\right)
$$

are Lipschitz with a uniform Lipschitz constant, say $K$, and these functions satisfy

$$
\left\|f_{j, \epsilon}^{+}-f_{j, \epsilon^{\prime}}^{+}\right\|_{\infty}<K\left|\epsilon-\epsilon^{\prime}\right|
$$


From this it follows that each $f_{j}^{+}$has a Lipschitz extension to $\overline{D^{+}}$. (The argument for $f_{j}^{-}$ is identical.) It then follows by continuity that

$$
f=\left\{f_{1}^{+}, \ldots, f_{d}^{+}\right\}=\left\{f_{1}^{-}, \ldots, f_{d}^{-}\right\} \text {on } \overline{D^{+}} \cap \overline{D^{-}}=\left\{\left(\xi^{\prime}, 0\right):\left\|\xi^{\prime}\right\|<1\right\} \stackrel{\text { def }}{=} H
$$

Now to show that $\partial \Gamma(f)=0$ in $D \times B$, we must show that $\Gamma(f)(d \beta)=0$ for all smooth $(n-1)$-forms $\beta$ with compact support in $D \times B$. Let $\beta$ be such a form and note that

$$
\begin{aligned}
\Gamma(f)(d \beta) & =\sum_{j=1}^{d} \int_{D^{+}} f_{j}^{+*} d \beta+\sum_{j=1}^{d} \int_{D^{-}} f_{j}^{-*} d \beta \\
& =\sum_{j=1}^{d} \int_{H} f_{j}^{+*} \beta-\sum_{j=1}^{d} \int_{H} f_{j}^{-*} \beta \\
& =0
\end{aligned}
$$

by (3.3.1). Passage to the second line of (3.3.2) amounts to the assertion that the boundary of the current defined by the graph of a Lipschitz function $\phi$ on a domain $\Omega$ with smooth boundary is the graph of $\phi$ restricted to $\partial \Omega$. Otherwise said: if $\Phi(x)=(x, \phi(x))$ is the graphing map, then $\partial \Phi_{*} \Omega=\Phi_{*} \partial \Omega$, an elementary fact from Geometric Measure Theory.

We have now proven that the support of $\partial \Gamma(f)$ is contained in the graph of $f$ restricted to a finite union of submanifolds of codimension $\geq 2$ in $A$. The $(n-1)$-dimensional integral geometric measure of this set is zero, and $\partial \Gamma(f)$ is a $(n-1)$-dimensional flat current. It therefore follows from $[\mathrm{Fe}, 4.1 .20]$ that $\partial \Gamma(f)=0$ as claimed.

Suppose now that $A$ is not smooth. Then the argument above proves that the support of $\partial \Gamma(f \circ p)$ is contained in the graph of $f \circ p$ restricted to $M-M^{\prime} \supset M-p^{-1}\left(s k_{m-2} A\right) \simeq$ $A-s k_{m-2} A$, a subcomplex of dimension $\leq m-2$. It therefore follows as in the preceeding paragraph that $\partial \Gamma(f)=p_{*}(\partial \Gamma(f \circ p))=0$.

Our next task is to verify that any Lipschitz map $f: A \rightarrow S P^{d}(B)$ can be approximated by good Lipschitz maps $f^{\prime}=\tilde{f} \circ \pi$.

Lemma 3.4. Let $U$ be a compact neighborhood of $S P^{d}(B)$ with retraction $\pi: U \rightarrow$ $S P^{d}(B)$ chosen as in Lemma 3.1 and equipped with a triangulation such that $\pi^{-1}(\Sigma)$ is a subcomplex of codimension $\geq 1$. Given a Lipschitz map $f: A \rightarrow S P^{d}(B)$ and $\epsilon>0$, there exists a good Lipschitz map $f^{\prime}=\pi \circ \tilde{f}: A \rightarrow U$ such that

(i) $\left\|f^{\prime}-f\right\|_{\infty}<\epsilon$,

(ii) $\operatorname{Lip}\left(f^{\prime}\right) \leq(1+\epsilon) \operatorname{Lip}(f)$.

Proof. Let $A \subset \mathbb{R}^{M}$ be a Lipschitz topological embedding which is a smooth immersion on $A_{\text {reg }}$ and restricts to a smooth map on each $m$-simplex of some triangulation of $A$. Extend $f$ to a neighborhood $V$ of $A$ using Kirszbraun's Theorem. Construct a smooth $\operatorname{map} g: V \rightarrow \mathbb{R}^{M}$ satisfying (i) and (ii) by the standard technique of convolution with an approximate identity (see [Ho, Chp 1]). Now by standard transversality theory (cf. $[\mathrm{A}-\mathrm{R}],[\mathrm{Hi}]), g$ can be approximated arbitrarily closely in $C^{1}$-norm by a smooth map whose restriction to every open simplex in $A$ is transversal to every open simplex in $\pi^{-1}(\Sigma)$.

The next proposition verifies the key step in the proof of the continuity of $\Gamma$ by relating the distance between good maps from $A$ to $S P^{d}(B)$ to the distance between their graphs. 
Proposition 3.5. Suppose $f_{0} \neq f_{1}: A \rightarrow S P^{d}(B)$ are good Lipschitz maps with $\operatorname{Lip}\left(f_{0}\right), \operatorname{Lip}\left(f_{1}\right) \leq K$. Then

$$
\left\|\Gamma\left(f_{1}\right)-\Gamma\left(f_{0}\right)\right\|_{b}<(1+c K)^{m}\left\|f_{1}-f_{0}\right\|_{\infty} .
$$

where $c$ is a constant depending only on the geometry of $S P^{d}(B)$.

Proof. As argued in the proof of Lemma 3.4, choose good maps $f_{i}^{\prime}=\pi \circ \tilde{f}_{i}$ with $\left\|f_{i}-f_{i}^{\prime}\right\|_{\infty}<$ $\left\|f_{1}-f_{0}\right\|_{\infty}$ and $\tilde{f}_{i}: A \rightarrow U$ transverse to $\pi^{-1}(\Sigma)$. Consider the homotopy

$$
\widetilde{h}(x, t)=(1-\varphi(t)) \widetilde{f}_{0}(x)+\varphi(t) \widetilde{f}_{1}(x)
$$

where $\varphi:[0,1] \rightarrow[0,1]$ is a smooth function with $\varphi(x)=0$ for $x \leq \frac{1}{8}, \varphi(x)=1$ for $x \geq \frac{7}{8}$, and $0 \leq \varphi^{\prime} \leq 2$. By transversality theory we perturb $\widetilde{h}: A \times I \rightarrow U$, keeping it fixed near 0 and 1 , so that it becomes transverse to $\pi^{-1}(\Sigma)$. This can be done arbitrarily closely in the $C^{1}$-norm. We now define $h=\pi \circ \widetilde{h}$ and note that $\operatorname{Lip}(h) \leq c K$, where $c$ depends only on the Lipschitz constant of $\pi: U \rightarrow S P^{d}(B)$ and the tolerance of approximation chosen for the perturbation.

If $H(x, t)=(x, t, h(x, t))$ is the graphing map, then one sees that the map $\Lambda^{m+1} H_{*}$ on $m+1$-currents induced by $H$ satisfies

$$
\left\|\Lambda^{m+1} H_{*}\right\|<(1+c K)^{m}\left\|f_{1}-f_{0}\right\|_{\infty} .
$$

Combining this with the arguments of [Fe, 4.1.14] and the proof of Theorem 2.4 gives us that $\operatorname{Mass}(\Gamma(h))<(1+c K)^{m}\left\|f_{1}-f_{0}\right\|_{\infty}$.

The arguments for Proposition 3.3 now prove that $\partial \Gamma(h)=0$ over $A \times(0,1)$. Furthermore, from the constancy of $\varphi$ near the boundary of $A \times[0,1]$ one sees directly that $\partial \Gamma(h)=\Gamma\left(f_{1}\right)-\Gamma\left(f_{0}\right)$. Using the definition of $\|-\|_{b}$ (cf. (1.0.1)), we conclude (3.5.1).

Although the graphing construction $\Gamma$ of Theorem 2.4 is not continuous on all Lipschitz maps $A \rightarrow S P^{d}(B)$, we show in the following theorem how to modify this construction to provide a continuous topological graphing map which agrees with $\Gamma$ on good Lipschitz maps.

Theorem 3.6. Let $A, B$ be compact pseudo-manifolds of dimension $m$ and $n$ respectively with $A$ oriented. There is a well-defined, continuous "topological graphing map"

$$
\Gamma^{\text {top }}: \operatorname{Map}_{\text {Lip }}\left(A, S P^{d}(B)\right) \rightarrow \mathcal{Z}_{m}(A \times B)
$$

which coincides with the graph mapping $\Gamma$ of Theorem 2.2 on the dense subset of good Lipschitz maps.

If $B$ is connected and provided with a choice of non-degenerate base point $b \in B$, then $\Gamma^{\text {top }}$ naturally extends to

$$
\operatorname{colim}_{d} \operatorname{Map}_{\text {Lip }}\left(A, S P^{d}(B)\right) \rightarrow \mathcal{Z}_{m}(A \times B) / \mathcal{Z}_{m}(A \times\{b\}),
$$

thereby determining a weak homotopy class of maps

$$
\Gamma^{\text {top }}: \operatorname{Map}_{\text {cont }}\left(A, \mathcal{Z}_{0}(B)^{o}\right) \rightarrow \mathcal{Z}_{m}(A \times B) / \mathcal{Z}_{m}(A \times\{b\}) .
$$


Proof. By Lemma 3.4, every $f \in \operatorname{Map}_{\text {Lip }}\left(A, S P^{d}(B)\right)$ is the uniform limit of a sequence $\left\{f_{k}\right\}_{k=1}^{\infty} \subset \operatorname{Map}_{\text {Lip }}\left(A, S P^{d}(B)\right)$ of good Lipschitz maps with uniformly bounded Lipschitz norm. From (2.4.1) we know that $\operatorname{Mass}\left(\Gamma\left(f_{k}\right)\right)$ of such a sequence $\left\{f_{k}\right\}_{k=1}^{\infty}$ is uniformly bounded. By Proposition 3.5, if $\left\{f_{k}\right\}_{k=1}^{\infty} \subset \operatorname{Map}_{\text {Lip }}\left(A, S P^{d}(B)\right)$ is a Cauchy sequence with uniformly bounded Lipschitz constant and if each $f_{k}$ is good, then there is a constant $K$ such that for all $k, \ell$,

$$
\left\|\Gamma\left(f_{k}\right)-\Gamma\left(f_{\ell}\right)\right\|_{b} \leq K\left\|f_{k}-f_{\ell}\right\|_{\infty} .
$$

Thus, $\left\{\Gamma\left(f_{k}\right)\right\}_{k=1}^{\infty}$ is a Cauchy sequence in $\mathcal{I}_{m}(A \times B)$. Now by [Fe;4.2.16] and [Fe;4.2.17] any sequence $\left\{\Gamma_{k}\right\}_{k=1}^{\infty}$ of integral $m$-currents on the compact polyhedron $A \times B$ which is Cauchy in the flat norm and for which $\operatorname{Mass}\left(\Gamma_{k}\right)+\operatorname{Mass}\left(\partial \Gamma_{k}\right)$ is uniformly bounded converges in $\mathcal{I}_{m}(A \times B)$ to an integral $m$-current on $A \times B$.

By Proposition 3.3, we have $\partial \Gamma\left(f_{k}\right)=0$ for all $k$. Since the subgroup $\mathcal{Z}_{m}(A \times B) \subset$ $\mathcal{I}_{m}(A \times B)$ of integral cycles is closed because $\partial$ is continuous on currents, the Cauchy sequence $\left\{\Gamma\left(f_{k}\right)\right\}_{k=1}^{\infty}$ converges to an integral cycle $\Gamma^{\text {top }}(f)$ on $A \times B$.

Consequently, we have extended $f \mapsto \Gamma(f)$ from the dense set of good maps to a continuous mapping

$$
\Gamma^{t o p}: \operatorname{Map}_{\text {Lip }}\left(A, S P^{d}(B)\right) \rightarrow \mathcal{Z}_{m}(A \times B)
$$

as claimed.

If $B$ is connected and provided with a non-degenerate base point $b \in B$, then the maps $\Gamma^{t o p}$ on $\operatorname{Map}_{\text {Lip }}\left(A, S P^{d}(B)\right)$ for varying $d$ are compatible when projected to $\mathcal{Z}_{m}(A \times$ $B) / \mathcal{Z}_{m}(A \times\{b\})$ and thereby determine the indicated map on colimits. Corollary 1.5 thus implies that $\Gamma^{\text {top }}$ determines the weak homotopy class of maps

$$
\Gamma^{\text {top }}: \operatorname{Map}_{\text {cont }}\left(A, \mathcal{Z}_{0}(B)^{o}\right) \rightarrow \mathcal{Z}_{m}(A \times B) / \mathcal{Z}_{m}(A \times\{b\}) .
$$

\section{§4. Slicing integral CURREnts}

Having constructed the topological graphing map

$$
\Gamma^{\text {top }}: \operatorname{Map}_{\text {Lip }}\left(A, \mathcal{Z}_{0}\left(S^{n}\right)\right) \rightarrow \mathcal{Z}_{m}\left(A \times S^{n}\right),
$$

we construct in this section a densely defined continuous "slicing map"

$$
\mathcal{Z}_{m}\left(A \times S^{n}\right) \rightarrow \mathcal{Z}_{m-n}(A)
$$

whose composition with $\Gamma^{\text {top }}$ will yield our topological duality map $\mathcal{D}^{\text {top }}$. This slicing is achieved by the Federer technique of slicing currents, which we now review.

Let $N$ be a smooth $n$-manifold and $y \in N$ some chosen point. Choose local coordinates $\left(\zeta_{1}, \ldots, \zeta_{n}\right)$ around $y$ (with $y$ corresponding to 0 ) and let

$$
\omega_{y, 1}=\phi(|\zeta|) d \zeta_{1} \wedge \cdots \wedge d \zeta_{n}
$$

where $\phi: \mathbb{R} \rightarrow \mathbb{R}$ is smooth, constant and positive near $0, \operatorname{supp}(\phi) \subset[-1,1]$, and $\int_{N} \omega_{y, 1}=$ 1. Set

$$
\omega_{y, \epsilon}=\rho_{\epsilon^{-1}}^{*} \omega_{y, 1}
$$

where $\rho_{t}: \mathbb{R}^{n} \rightarrow \mathbb{R}^{n}$ is scalar multiplication by $t$.

The following theorem recalls some basic properties of Federer's slicing theory, specialized to the case of products. 
Theorem 4.1. [Fe, 4.3] Let $A$ be a compact oriented pseudo-manifold and $N$ a smooth, closed manifold of dimension $n$. Suppose $T \in \mathcal{I}_{m}(A \times N)$ is an integral current of dimension $m \geq n$. Then for almost all $y \in N$ the limit

$$
\operatorname{sl}_{y}(T)=\lim _{\epsilon \rightarrow 0^{+}} T \wedge p r_{N}^{*}\left(\omega_{y, \epsilon}\right)
$$

exists in the flat topology. Furthermore, $\mathrm{sl}_{y}(T)$ is an integral current of dimension $m-n$ on $A \times N$ and

$$
\partial \mathrm{sl}_{y}(T)=\operatorname{sl}_{y}(\partial T)
$$

Note. We recall that for a smooth $n$-form $\omega$, the current $T \wedge \omega$ is defined on a smooth $(m-n)$-form $\delta$ by $(T \wedge \omega)(\delta)=T(\omega \wedge \delta)$.

Let $\operatorname{pr}_{A}: A \times N \rightarrow A$ denote the projection map, and for $y, T$ as above set

$$
\mathrm{Sl}_{y}(T) \equiv\left(\operatorname{pr}_{A}\right)_{*}\left(\operatorname{sl}_{y}(T)\right)
$$

Then by Theorem 4.1 we have that

$$
\mathrm{Sl}_{y}(T) \in \mathcal{I}_{m-n}(A) \quad \text { and } \quad \partial \mathrm{Sl}_{y}(T)=\mathrm{Sl}_{y}(\partial T)
$$

Moreover, for any $R \in \mathcal{I}(A)$ and any $y \in N$,

$$
\mathrm{Sl}_{y}(R \times[N])=R
$$

As a consequence of Theorem 4.1, we conclude that Federer slicing determines a map on integral homology. We recall (cf. [Fe, 4.4.1]) that the integral cohomology of $A$ can be computed as the homology of the chain complex of integral currents on $A$,

$$
H_{i}(A ; \mathbb{Z})=H_{i}\left(\mathcal{I}_{*}(A)\right), \quad i \geq 0
$$

Corollary 4.2. Suppose $T \in \mathcal{I}_{m}(A \times N)$ and $\partial T=0$ (i.e., $T \in \mathcal{Z}_{m}(A \times N)$ ). Then for almost all $y \in N$ the class of $\operatorname{Sl}_{y}(T)$ in $H_{m-n}\left(\mathcal{I}_{*}(A)\right) \cong H_{m-n}(A ; \mathbb{Z})$ is independent of $y$ and depends only on the homology class of $T$. Thus slicing induces a well-defined homomorphism

$$
\mathrm{Sl}: H_{m}(A \times N ; \mathbb{Z}) \rightarrow H_{m-n}(A ; \mathbb{Z})
$$

left inverse to external multiplication by the fundamental class $[N] \in H_{n}(N ; \mathbb{Z})$. Furthermore, for any chosen base point $y_{0} \in N$ this map factors naturally through a homomorphism

$$
\mathrm{Sl}: H_{m}\left(A \times N, A \times\left\{y_{0}\right\} ; \mathbb{Z}\right) \rightarrow H_{m-n}(A ; \mathbb{Z})
$$

Proof. If $T=\partial S$ where $S$ is rectifiable, then $S l_{y}(T)=\partial\left(S l_{y}(S)\right)$ for almost all $y$ by (4.1.1). Hence, $\left[S l_{y}(T)\right] \in H_{m-n}(A ; \mathbb{Z})$ depends only on the class $[T] \in H_{m}(A \times N ; \mathbb{Z})$. 
To prove that $S l_{y}(T)$ is homologous to $S l_{y^{\prime}}(T)$ for almost all $y, y^{\prime} \in N$, we work locally on $N$ and assume that $y$ and $y^{\prime}$ lie in a small coordinate ball $B$. Let $F: N \times[0,1] \rightarrow N$ be an isotopy supported in $B$ with $F_{0}=$ Id and with $F_{1}(y)=y^{\prime}$. Extend $F$ trivially to $\widetilde{F}$ : $A \times N \times[0,1] \rightarrow A \times N$. Applying [Fe, 4.3.9] gives a current $R$ with $\partial R=s l_{y}(T)-s l_{y^{\prime}}(T)$. Thus, the class of $s_{y}(T)$ is independent of $y \in N$. The fact that Federer slicing is left inverse to external multiplication by $[N]$ is immediate from (4.1.3).

For the final assertion, observe for any integral current $T$ with support in $A \times\left\{y_{0}\right\}$ that the slice $\operatorname{sl}_{y}(T)=0$ for all $y \neq y_{0}$. Hence, our construction can be carried out on the relative chain complex $\mathcal{I}_{*}(A \times N) / \mathcal{I}_{*}\left(A \times\left\{y_{0}\right\}\right)$

In the next Proposition we explicitly compute $\operatorname{sl}_{y}(T)\left(\Gamma^{t o p}(f)\right)$ for suitably chosen representatives $f: A \rightarrow S P^{d}(N)$ of $\pi_{0} \operatorname{Map}_{\text {cont }}\left(A, S P^{d}(N)\right)$ and points $y \in N$ regular for the lifts $f_{\tau, 1}, \ldots, f_{\tau, k(\tau)}: \tau \rightarrow N$ of the restrictions $\left.f\right|_{\tau}$.

We say that a smooth map $g: \Delta^{m} \rightarrow \Delta^{n}$ between closed simplices has $y \in \operatorname{interior}\left(\Delta^{n}\right)$ as a regular value if $y$ is a regular value of $\left.f\right|_{D}$ for every open face $D$ (of dimension $\leq m$ ) of $\Delta$.

Under this hypothesis either $g^{-1}(y)=\emptyset$ or $g^{-1}(y)$ is a smooth submanifold of codimension$n$ in $\Delta^{m}$ which meets every face of $\partial \Delta^{m}$ transversely. In this latter case there is also a closed disk neighborhood $y \in D^{n} \subset \operatorname{interior}\left(\Delta^{n}\right)$ and a diffeomorphism

$$
g^{-1}\left(D^{n}\right) \cong g^{-1}(y) \times D^{m}
$$

with respect to which the restriction of $g$ becomes projection onto the second factor.

Proposition 4.3. Let $A$ be a compact oriented pseudo-manifold of dimension $m$ and $N$ a smooth closed oriented manifold of dimension $n$. Let $f: A \rightarrow S P^{d}(N)$ be a Lipschitz map such that $A, S P^{d}(N)$, and $N$ admit triangulations $\mathcal{T}, \mathcal{R}$, and $\mathcal{S}$ respectively with the following properties:

(1) $\mathcal{R}$ is subordinate to the singular stratification of $S P^{d}(N)$;

(2) if $\tilde{\mathcal{R}}$ denotes the equivariant triangulation of $N^{\times d}$ induced by $\mathcal{R}$ on $S P^{d}(N)$, then the projections $\mathrm{pr}_{i}$ of each closed simplex in $\tilde{\mathcal{R}}$ are subcomplexes of $N$;

(3) for any open $n$-simplex $\delta \in \mathcal{S}(n)$, almost every point of $\delta$ is a regular value for every $f_{\tau, i}$, where $\times_{i} f_{\tau, i}: \tau \rightarrow N^{\times d}$ denotes the lifting of $f$ restricted to an open simplex $\tau \in \mathcal{T}$.

Then for almost every point $y$ of an open $n$-simplex $\delta \in \mathcal{S}(n), \mathrm{sl}_{y}(\Gamma(f))$ is well defined and

$$
\mathrm{Sl}_{y}(\Gamma(f))=\sum_{\tau \in \mathcal{T}(m)} \sum_{i=1}^{k(\tau)} d_{i}^{\tau} \cdot\left[f_{\tau, i}^{-1}(y)\right]
$$

where $\left[f_{i, \tau}^{-1}(y)\right]$ is the integral $(m-n)$-current on $\tau \subset A$ associated to the submanifold $f_{i, \tau}^{-1}(y)$ oriented by the orientations of $A$ and $N$ and $\left\{d_{1}^{\tau}, \ldots, d_{k(\tau)}^{\tau}\right\}$ are the multiplicities associated to the open stratum of $S P^{d}(N)$ containing $f(\tau)$.

Proof. To verify (4.3.1), we must show

$$
\lim _{\epsilon \rightarrow 0} \Gamma(f)\left(\operatorname{pr}_{A}^{*} \psi \wedge \operatorname{pr}_{N}^{*}\left(\omega_{y, \epsilon}\right)\right)=\sum_{\tau \in \mathcal{T}(m)} \sum_{i=1}^{k(\tau)} d_{i}^{\tau} \int_{\left[f_{\tau, i}^{-1}(y)\right]} \psi
$$


for all smooth $(m-n)$-forms $\psi$ on $A$. In view of the definition of $\Gamma(f)$ (cf. Theorem 2.4), it suffices to verify for all differential $(m-n)$-forms $\psi$, all simplices $\tau \in \mathcal{T}(m)$, and all $i$ the equality

$$
\lim _{\epsilon \rightarrow 0} G\left(f_{\tau, i}\right)\left(\psi \wedge \operatorname{pr}_{N}^{*}\left(\omega_{y, \epsilon}\right)\right)=\int_{\left[f_{\tau, i}^{-1}(y)\right]} \psi
$$

where $G(f)$ denotes the usual graph of a smooth map $f$.

By assumption almost every point $y$ in an $n$-simplex $\delta$ of $N$ is a regular value for all maps $f_{\tau, i}$. Choosing such a $y$ and applying the local product structure (4.2.2), one easily establishes (4.3.3).

Proposition 4.4. Let $A$ be a compact oriented pseudo-manifold of dimension $m$ and $N$ a smooth closed manifold of dimension $n \leq m$. Then every homotopy class of continuous maps from $A$ to $S P^{d}(N)$ contains a representative $f: A \rightarrow S P^{d}(N)$ which admits triangulations satisfying the conditions of Proposition 4.3.

Moreover, if $A=X, N=Y$ are complex projective algebraic varieties of dimension $d, e$ respectively with $Y$ and if $f: X \rightarrow Y$ is a morphism, then $f^{a n}: X^{a n} \rightarrow S P^{d}\left(Y^{a n}\right)$ admits such a triangulation.

Note. Here and below the superscript "an" denotes the underlying analytic structure of the algebraic object.

Proof. Choose a triangulation of $S P^{d}(N)$ subordinate to the singular stratification, thereby

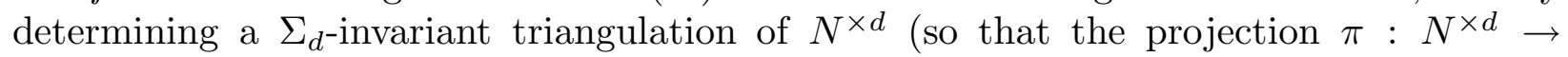
$S P^{d}(N)$ restricts to a homeomorphism on every open simplex of $N^{\times d}$ ). Choose a triangulation of $N$ with the property that images $\operatorname{pr}_{i}(\bar{\sigma})$ of all closed simplices $\bar{\sigma}$ of $N^{\times d}$ (with respect to the just constructed $\Sigma_{d}$-invariant triangulation of $N^{\times d}$ ) are subcomplexes of $N$ for each projection $\mathrm{pr}_{i}: N^{\times d} \rightarrow N$. Thus, conditions 1.) and 2.) of Proposition 4.3 are satisfied. Observe that if $y \in N$ is an interior point of any $n$-simplex of $N$ so triangulated, then

$$
y \notin \bigcup_{i=1}^{d} \operatorname{pr}_{i}\left(s k_{n-1}\left(N^{\times d}\right)\right)
$$

where $s k_{n-1}(-)$ denotes the $(n-1)$-skeleton functor.

Let $g: A \rightarrow S P^{d}(N)$ be a Lipschitz map. Then by standard techniques we can choose a triangulation of $A$ and an approximation $f$ of $g$ which is a simplicial mapping. In particular $f$ is homotopic to $g$, and for each simplex $\tau$ in $A, f$ restricts to be a smooth mapping $f: \tau \rightarrow \Delta^{n}$ for some $n$-simplex $\Delta^{n}$ of $N$. Assertion (3) now follows from Sard's Theorem.

If $f: X \rightarrow Y$ is a smooth morphism, then $f$ is smooth when restricted to some Zariski open subset of $X$. If we choose the triangulations as above with the additional conditions that the triangulations of $X, Y$ are such that the singular loci are subcomplexes, then once again almost every interior point $y \in Y$ of a $2 e$-simplex of $N$ is a regular value for each $f_{\tau, i}$ for each $2 d$-simplex $\tau$ of $X$ and each $i, 1 \leq i \leq d$. 


\section{§5. Poincaré duality}

In this section, we verify that $\Gamma^{t o p}$ of Theorem 3.6 induces the usual Poincaré duality map (i.e., cap product with the fundamental class) for closed, oriented pseudo-manifolds of dimension $m$. This identification is achieved by first giving a concrete realization of the equivalence $H^{n}(A, \mathbb{Z}) \simeq \pi_{0} \operatorname{Map}_{\text {cont }}\left(A, Z_{0}\left(S^{n}\right)^{o}\right)$ in terms of "integral" differential forms on $S P^{d}\left(S^{n}\right)$ constructed in Proposition 5.1. Using an explicit cocycle to represent a class in $H^{n}(A, \mathbb{Z})$, we then verify that the cap product of this cocycle with the fundamental class of $A$ yields a cycle homologous to the integral cycle obtained in Proposition 4.3 as the slice of the topological graph of the map given by this concrete realization. This verifies that $\pi_{0}$ applied to our topological duality map $\mathcal{D}^{\text {top }}$ (i.e., topological graphing followed by current slicing) is the Poincaré duality map defined as cap product with the fundamental class. The general case of identifying the map on $\pi_{j}$ induced by $\mathcal{D}^{\text {top }}$ is then proved using a formal dimension-shifting argument.

We begin with a brief discussion of de Rham theory on a finite simplicial complex $\mathcal{S}$. By a differential $k$-form on $\mathcal{S}$ we mean an assignment of a smooth $k$-form $\varphi_{\sigma} \in \mathcal{E}^{k}(\sigma)$ on each closed simplex $\sigma$ in $\mathcal{S}$ with the property that whenever $\tau \subset \sigma$ is a face of $\sigma$, one has $\left.\varphi_{\sigma}\right|_{\tau}=\varphi_{\tau}$. Under the de Rham differential, the space of such forms becomes a complex which computes the real cohomology of $|\mathcal{S}|$. (See $[\mathrm{G}-\mathrm{M}]$ for example.)

If $\varphi$ is a closed $k$-form on $\mathcal{S}$ with the property that $\int_{\sigma} \varphi \in \mathbb{Z}$ for every closed $k$-simplex in $\mathcal{S}$, then $\varphi$ defines an integral simplicial cocycle on $\mathcal{S}$ and thereby determines a class $[\varphi] \in H^{k}(|\mathcal{S}|, \mathbb{Z})$.

Proposition 5.1. Choose a triangulation $\mathcal{R}$ of $S P^{d}\left(S^{n}\right)$ subordinate to the singular stratification and let $\tilde{\mathcal{R}}$ denote the associated triangulation of $\left(S^{n}\right)^{\times d}$; choose a triangulation $\mathcal{S}$ of $S^{n}$ so that $p r_{i}$ for each $i, 1 \leq i \leq d$, applied to each closed simplex of $\tilde{\mathcal{R}}$ is a subcomplex of $\mathcal{S}$. Let $y \in S^{n}$ be a point of some open $n$-simplex of $\mathcal{S}$, so that $y$ satisfies (4.4.1). Finally, choose $\epsilon>0$ such that

$$
\operatorname{supp}\left(\omega_{y, \epsilon}\right) \cap \bigcup_{i=1}^{d} \operatorname{pr}_{i}\left(s k_{n-1}\left(\left(S^{n}\right)^{\times d}\right)\right)=\emptyset
$$

where $\omega_{y, \epsilon}$ is given by (4.0). Then for all $\epsilon$ sufficiently small, there exists a closed differential $n$-form $\Omega_{y, \epsilon}$ on the triangulation $\mathcal{R}$ of $S P^{d}\left(S^{n}\right)$, uniquely determined by the condition that

$$
\pi^{*}\left(\Omega_{y, \epsilon}\right)=\sum_{i=1}^{d} \operatorname{pr}_{i}^{*}\left(\omega_{y, \epsilon}\right)
$$

where $\pi:\left(S^{n}\right)^{\times d} \rightarrow S P^{d}\left(S^{n}\right)$ is the quotient map and $\operatorname{pr}_{i}:\left(S^{n}\right)^{\times d} \rightarrow S^{n}$ are the projections.

This form $\Omega_{y, \epsilon}$ is compactly supported in the complement of the $(n-1)$-skeleton, and satisfies $\int_{\sigma} \Omega_{y, \epsilon} \in \mathbb{Z}$ for all $n$-simplices $\sigma$. Thus, as above, $\Omega_{y, \epsilon}$ represents an integral simplicial cocycle on $S P^{d}\left(S^{n}\right)$ with respect to the triangulation $\mathcal{R}$. 
Moreover, if $j: S^{n} \rightarrow S P^{d}\left(S^{n}\right)$ is the map sending $v \in S^{n}$ to $(v, y, \ldots, y) \in S P^{d}\left(S^{n}\right)$ and if $j_{*}\left[S^{n}\right]$ denotes the integral $n$-current on $S P^{d}\left(S^{n}\right)$ given as the image of the fundamental class of $S^{n}$, then

$$
j_{*}\left[S^{n}\right]\left(\Omega_{y, \epsilon}\right)=\int_{S^{n}} j^{*}\left(\Omega_{y, \epsilon}\right)=1
$$

Proof. For each open simplex $\sigma$ of $S P^{d}\left(S^{n}\right), \pi^{-1}(\sigma)$ is the disjoint union of some number (between 1 and $d$ !) of open simplices $\tilde{\sigma} \subset\left(S^{n}\right)^{\times d}$ each mapping homeomorphically to $\sigma$. Thus, (5.1.2) necessarily characterizes $\Omega_{y, \epsilon}$. On the other hand, since $\sum_{i=1}^{d} \operatorname{pr}_{i}^{*}\left(\omega_{y, \epsilon}\right)$ is $\Sigma_{d}$-equivariant, we may define $\Omega_{y, \epsilon}$ satisfying (5.1.2) on an open simplex $\sigma$ of $S P^{d}\left(S^{n}\right)$ by setting

$$
\left.\left.\left(\Omega_{y, \epsilon}\right)\right|_{\sigma} \equiv\left(\sum_{i=1}^{d} \operatorname{pr}_{i}^{*}\left(\omega_{y, \epsilon}\right)\right)\right|_{\tilde{\sigma}}
$$

where $\tilde{\sigma}$ is any open simplex of $\left(S^{n}\right)^{\times d}$ mapping homeomorphically onto an open simplex $\sigma$ of $S P^{d}\left(S^{n}\right)$. Since $\sum_{i=1}^{d} \operatorname{pr}_{i}^{*}\left(\omega_{y, \epsilon}\right)$ is a closed $n$-form on $\left(S^{n}\right)^{\times d}$, it is easily checked that $\Omega_{y, \epsilon}$ is $d$-closed.

Observe that (5.1.1) implies that the restriction of $\Omega_{y, \epsilon}$ to any closed $n$-simplex $\bar{\sigma}$ has support in $\sigma$. Therefore,

$$
\Omega_{y, \epsilon}(\bar{\sigma})=\sum_{i=1}^{d} \int_{\tilde{\sigma}} \operatorname{pr}_{i}^{*}\left(\omega_{y, \epsilon}\right)=\sum_{i=1}^{d} \operatorname{deg}\left(\left.\operatorname{pr}_{i}\right|_{\tilde{\sigma}}\right) \cdot \int_{S^{n}} \omega_{y, \epsilon} \in \mathbb{Z}
$$

where $\operatorname{deg}\left(\left.\operatorname{pr}_{i}\right|_{\tilde{\sigma}}\right)$ is the degree of the map from $(\overline{\tilde{\sigma}}, \overline{\tilde{\sigma}}-\tilde{\sigma})$ to $\left(S^{n}, S^{n}-\operatorname{supp}\left(\omega_{y, \epsilon}\right)\right)$ given by the restriction of $\operatorname{pr}_{i}$.

Finally,

$$
\Omega_{y, \epsilon}\left(j_{*}\left[S^{n}\right]\right)=\int_{\left[\left(S^{n}, y, y, \ldots, y\right)\right]} \sum_{i=1}^{d} \operatorname{pr}_{i}^{*}\left(\omega_{y, \epsilon}\right)=\int_{S^{n}} \omega_{y, \epsilon}=1
$$

Proposition 5.1 enables us to give an explicit representation of integral cohomology classes as pull-backs of the integral form $\Omega_{y, \epsilon}$.

Proposition 5.2. Let $A$ be a compact oriented pseudo-manifold of dimension $m$. Then the natural identification of $H^{n-j}(A, \mathbb{Z})$ with homotopy classes of maps into the EilenbergMacLane space $K(\mathbb{Z}, n)$,

$$
\theta: \pi_{j} \operatorname{Map}_{\text {cont }}\left(A, \mathcal{Z}_{0}\left(S^{n}\right)^{o}\right) \stackrel{\simeq}{\longrightarrow} H^{n-j}(A, \mathbb{Z}),
$$

can be realized as follows. Choose triangulations $\mathcal{R}, \mathcal{S}$ of $S P^{d}(n), S^{n}$ respectively; choose $\epsilon>0$ so that (5.1.1) is satisfied. For $\gamma \in \pi_{j} \operatorname{Map}_{\text {cont }}\left(A, \mathbb{Z}_{0}\left(S^{n}\right)^{o}\right)$, choose a triangulation of $S^{j} \times A$ and a simplicial map $g: S^{j} \times A \rightarrow S^{j} \wedge A \rightarrow S P^{d}\left(S^{n}\right)$ representing $\gamma$. Then $\theta(\gamma)$ 
equals the Künneth projection $H^{n}\left(A \times S^{j}, \mathbb{Z}\right) \rightarrow H^{n-j}(A, \mathbb{Z})$ applied to the cohomology class of the simplicial integral cocycle $g^{*}\left(\Omega_{y, \epsilon}\right)$ on $A \times S^{j}$,

Proof. Using the simplicial approximation thoerem, we see that any element $\gamma \in$ $\pi_{j} \operatorname{Map}_{\text {cont }}\left(A, \mathbb{Z}_{0}\left(S^{n}\right)^{o}\right)$ can be represented by a simplicial map $f: A \times S^{j} \rightarrow S P^{d}\left(S^{n}\right)$ for $d$ sufficiently large (which sends the wedge $A \vee S^{j}$ to the base point of $S^{n}$ ). The identification of (5.2.1) is given by restricting the fundamental class $\iota_{n} \in H^{n}(K(\mathbb{Z}, n), \mathbb{Z})=$ $H^{n}\left(S P^{\infty}\left(S^{n}\right), \mathbb{Z}\right)$ to $H^{n}\left(S P^{d}\left(S^{n}\right), \mathbb{Z}\right)$, then applying $f^{*}: H^{n}\left(S P^{d}\left(S^{n}\right), \mathbb{Z}\right) \rightarrow H^{n}(A \times$ $\left.S^{j}, \mathbb{Z}\right)$, and finally applying the Künneth theorem.

Since the natural maps $H^{n}\left(S P^{\infty}\left(S^{n}\right), \mathbb{Z}\right) \rightarrow H^{n}\left(S P^{d}\left(S^{n}\right), \mathbb{Z}\right) \rightarrow H^{n}\left(S^{n}, \mathbb{Z}\right)$ are isomorphisms (cf. [Mi]), we conclude using Proposition 5.1 that the restriction of $\iota_{n}$ to $H^{n}\left(S P^{d}\left(S^{n}\right), \mathbb{Z}\right)$ equals the cohomology class of the simplicial integral cocycle $\Omega_{y, \epsilon}$ on $S P^{d}\left(S^{n}\right)$. Since $g$ is simplicial, $g^{*}\left(\Omega_{y, \epsilon}\right)$ takes integral values on $n$-simplices of $A \times S^{j}$ (as argued for $\Omega_{y, \epsilon}$ in the proof of Proposition 5.1) and thus has cohomology class equal to the pull-back via $g^{*}$ of the cohomology class of $\Omega_{y, \epsilon}$.

As we see below, Propositions 4.3 and 5.2 enable us to identify $\mathcal{A} \circ \Gamma^{\text {top }}$ on $\pi_{0} \operatorname{Map}_{\text {cont }}\left(A, \mathbb{Z}_{0}\left(S^{n}\right)^{o}\right)$.

Theorem 5.3. Let $A$ be a compact oriented pseudo-manifold of dimension $m$. Then the following diagram commutes

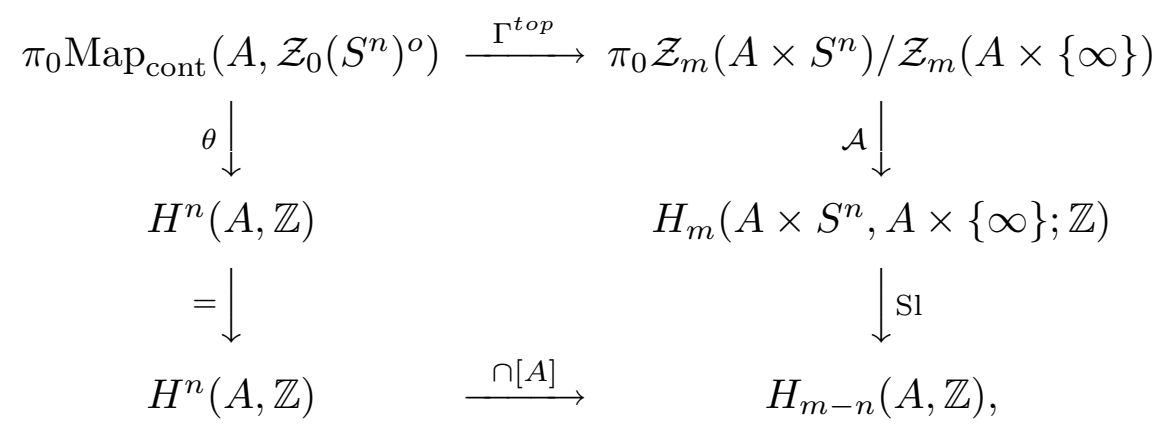

where $\theta$ is the isomorphism of Proposition 5.2, $\mathcal{A}$ is the Almgren isomorphism of Theorem 1.2 , and $\mathrm{Sl}$ is the homomorphism of Corollary 4.2 associated to Federer slicing of currents (which is an isomorphism in this special case for which $N$ is a sphere).

Proof. We restrict attention to a given homotopy class of maps from $A$ to $S P^{d}\left(S^{n}\right)$ representing a class in $\pi_{0} \operatorname{Map}_{\text {cont }}\left(A, \mathcal{Z}_{0}\left(S^{n}\right)^{o}\right)$. Choose a triangulation $\mathcal{S}$ of $S P^{d}\left(S^{n}\right)$ subordinate to the singular stratification and a triangulation $\mathcal{T}$ of $A$ as in Proposition 4.3. Let $f: A \rightarrow S P^{d}\left(S^{n}\right)$ be a simplicial representative of our homotopy class. Let $\mathcal{U}$ be a triangulation of $S^{n}$ such that each projection $\mathrm{pr}_{i}$ maps simplices of $S^{n \times d}$ (with triangulation induced by $\mathcal{S}$ ) to subcomplexes of $\mathcal{U}$. Replacing these triangulations by their barycentric subdivisions if necessary, we may assume that there is a closed $n$-simplex $\bar{\sigma} \subset S^{n}$ with both barycenter $z$ and last indexed vertex $y$ regular values. Choose $\epsilon>0$ so that the support of $\omega_{z, \epsilon} \subset \sigma$.

If $f$ is a suitable simplicial representative of a class in $\pi_{0} \operatorname{Map}_{\text {cont }}\left(A, \mathcal{Z}_{0}\left(S^{n}\right)^{o}\right)$, then $f^{*}\left(\Omega_{z, \epsilon}\right)$ represents the cohomology class $\theta(f)$. Observe that for any (closed) $n$-simplex $\mu$ 
of $\mathcal{T}$

$$
\int_{\mu} f^{*}\left(\Omega_{z, \epsilon}\right)=\sum_{i=1}^{k(\mu)} d_{i}^{\mu} \cdot \int_{\mu} f_{\mu, i}^{*}\left(\omega_{z, \epsilon}\right)
$$

We choose an ordering on the vertices of $A$ and employ the classical combinatorial formulation of cap product (cf. [H-Y]),

$$
f^{*}\left(\Omega_{z, \epsilon}\right) \cap[A]=\sum_{\tau \in \mathcal{T}(m)}\left\{\int_{\bar{\tau}_{f}} f^{*}\left(\Omega_{z, \epsilon}\right)\right\} \cdot \bar{\tau}_{b}
$$

which equals

$$
\sum_{\tau \in \mathcal{T}(m)} \sum_{i=1}^{k(\tau)} d_{i}^{\tau} \cdot\left\{\int_{\bar{\tau}_{f}} f_{\tau, i}^{*}\left(\omega_{z, \epsilon}\right)\right\} \cdot \bar{\tau}_{b}
$$

where $\bar{\tau}_{f}, \bar{\tau}_{b}$ are the front $n$-face, back $(m-n)$-face of $\bar{\tau}$.

By construction, $f_{\tau, i}^{-1}(y)$ is a $(m-n)$-subcomplex of $\bar{\tau}$ and $f_{\tau, i}^{*}\left(\omega_{z, \epsilon}\right)$ is a non-vanishing $n$ form on $\bar{\tau}_{f}$ if and only if $\bar{\tau}_{f}$ projects onto $\bar{\sigma}$ which is the case if and only if $\bar{\tau}$ projects onto $\bar{\sigma}$ and $\bar{\tau}_{b}$ is a top dimensional simplex of $f_{\tau, i}^{-1}(y)$. Replacing $f$ by its barycentric subdivision if necessary so that the vertex $y$ is regular and is the barycenter of a top dimensional simplex of a triangulation $\mathcal{U}$ of $S^{n}$ as above, we may arrange that the condition that $\bar{\tau}_{b}$ is a top dimensional simplex of $f_{\tau, i}^{-1}(y)$ implies $\bar{\tau}$ projects onto $\bar{\sigma}$. In other words,

$$
\int_{\bar{\tau}_{f}} f_{\tau, i}^{*}\left(\omega_{y, \epsilon}\right)=1 \Longleftrightarrow f_{\tau, i}\left(\bar{\tau}_{f}\right)=\bar{\sigma} \Longleftrightarrow \bar{\tau}_{b} \text { is an } m-n \text {-simplex of } f_{\tau, i}^{-1}(y)
$$

and $\int_{\bar{\tau}_{f}} f_{\tau, i}^{*}\left(\omega_{y, \epsilon}\right)$ equals 0 otherwise.

In other words, the simplicial chain (5.3.2) equals the integral current $\mathrm{Sl}_{y}(\Gamma(f))$ of (4.3.1).

Recall that the Almgren isomorphism restricted to connected components of spaces of integral cycles $\mathcal{A}: \pi_{0} \mathcal{Z}_{m}\left(A \times S^{n}\right) \simeq H_{m}\left(A \times S^{n} ; \mathbb{Z}\right)$ is given by the natural identification of the $m$ th homology group of the complex $I_{*}\left(A \times S^{n}\right)$ with the homology group $H_{m}\left(A \times S^{n} ; \mathbb{Z}\right)$ as constructed by Federer in [Fe] and used in Corollary 4.2. Thus, Corollary 4.2 together with Proposition 4.4 (which gives us (4.3.1)) implies that

$$
\theta([f]) \cap[A]=f^{*}\left(\Omega_{x, \epsilon}\right) \cap[A]=(\mathrm{Sl} \circ \mathcal{A})\left(\Gamma^{t o p}(f)\right)
$$

This establishes the commutativity of (5.3.1).

Theorem 5.3 leads us to the general identification (i.e., on all homotopy groups) of $\Gamma_{*}^{t o p}$ with the Poincaré duality map. 
Theorem 5.4. Let $A$ be a compact oriented pseudo-manifold of dimension $m$. Then for any $j, 0 \leq j \leq n$, the following diagram commutes

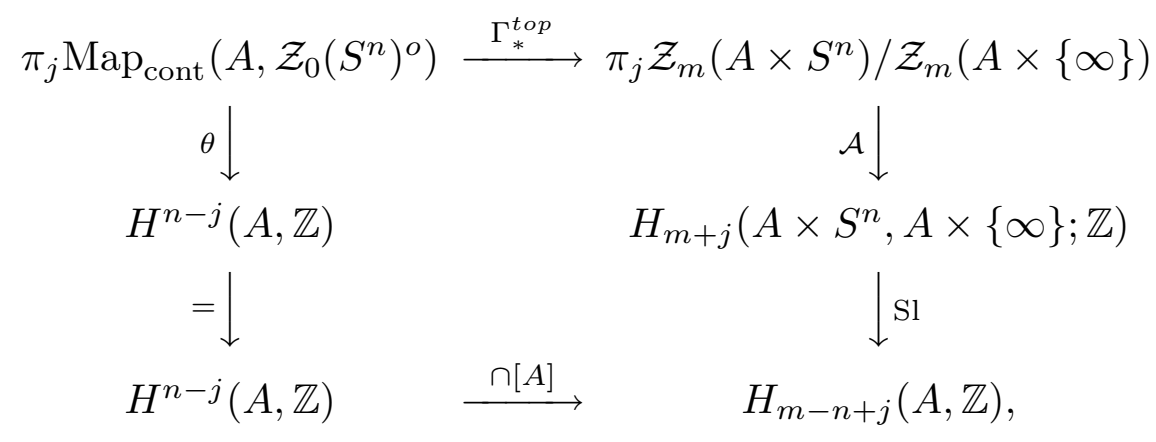

where $\theta$ is the isomorphism of Proposition 5.2, $\mathcal{A}$ is the Almgren isomorphism of Theorem 1.2, and $\mathrm{Sl}$ is the homomorphism of Corollary 4.2 associated to Federer slicing of currents.

Proof. We proceed by induction on $j$ : for each $j \geq 0$, we establish the commutativity of (5.4.1) for all $n \geq 0$. (In the degenerate case $n=0$, we set $\mathcal{Z}_{0}\left(S^{0}\right)^{o}$ equal to $\mathbb{Z}$ with the discrete topology.) The case $j=0$ is the conclusion of Theorem 5.3 ; for $j>0$, the case $n=0$ is immediate.

Consider the commutative diagram for some $n>0$ :

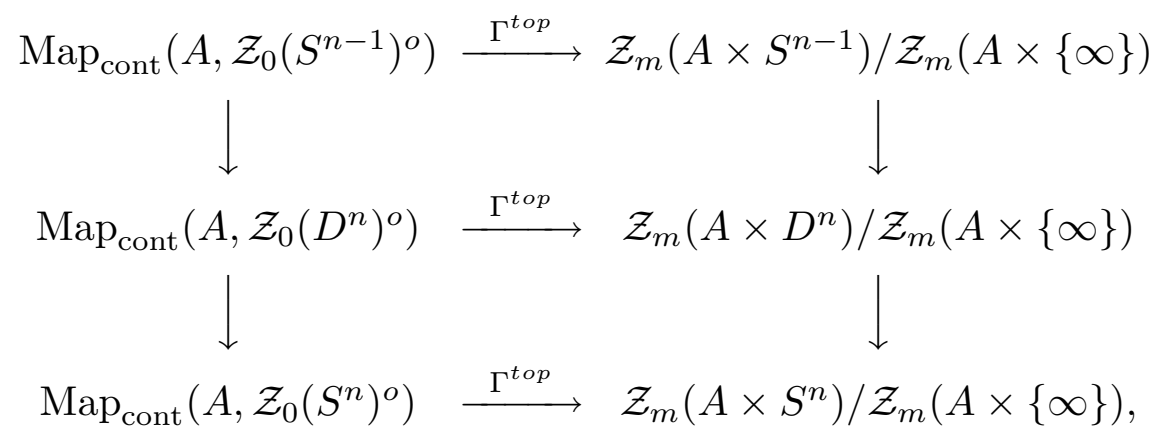

where $S^{n-1} \subset D^{n} \subset S^{n}$ is the inclusion of the $n-1$-sphere as the boundary of the closed $n$-disk which is then collapsed to become the base point of $S^{n}$. The Dold-Thom Theorem [D-T] enables us to conclude that the triple $\mathcal{Z}_{0}\left(S^{n-1}\right)^{o} \rightarrow \mathcal{Z}_{0}\left(D^{n}\right)^{o} \rightarrow \mathcal{Z}_{0}\left(S^{n}\right)^{o}$ is a fibration sequence (cf. [F-G]), so that the left vertical row of (5.4.2) is also a fibration sequence. Moreover, Theorem 1.2 implies that the right vertical row of (5.4.2) is also a fibration sequence. Observe that $\operatorname{Map}_{\text {cont }}\left(A, \mathcal{Z}_{0}\left(D^{n}\right)^{o}\right)$ is contractible, whereas $\mathcal{Z}_{m}(A \times$ $\left.D^{n}\right) / \mathcal{Z}_{m}(A \times\{\infty\})$ is contractible by Theorem 1.2 .

Consequently, the naturality of the boundary map in the long exact homotopy sequence for a fibration sequence implies the commutativity of the square

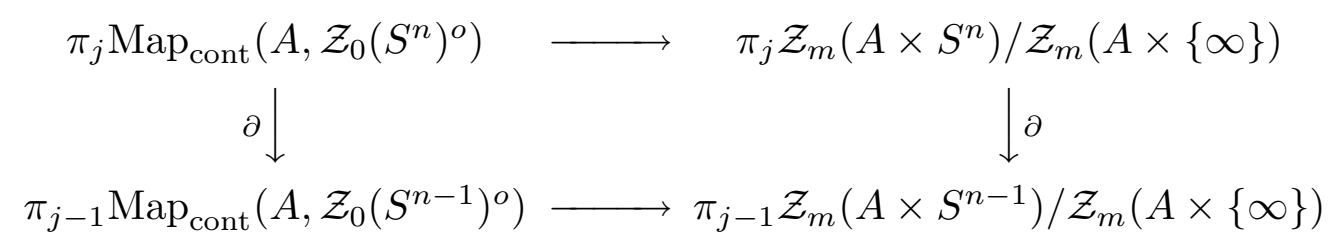

whose vertical maps are isomorphisms. 
Thus, induction on $j$ will complete the proof of the theorem once we verify that

$$
\theta \circ \partial=\theta: \pi_{j} \operatorname{Map}_{\text {cont }}\left(A, \mathcal{Z}_{0}\left(S^{n}\right)^{o}\right) \rightarrow H^{n-j}(A, \mathbb{Z})
$$

and

$$
\mathrm{Sl} \circ \mathcal{A} \circ \partial=\mathrm{Sl} \circ \mathcal{A}: \pi_{j}\left\{\mathcal{Z}_{m}\left(A \times S^{n}\right) / \mathcal{Z}_{m}(A \times\{\infty\})\right\} \rightarrow H_{m-n+j}(A)
$$

We easily verify the commutativity of the square

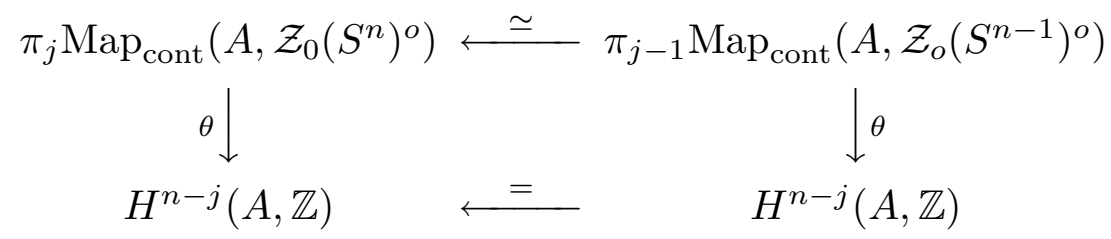

where the upper horizontal arrow is induced by the equivalence $\mathcal{Z}_{0}\left(S^{n-1}\right) \rightarrow \Omega \mathcal{Z}_{0}\left(S^{n}\right)$ associated to $\Sigma S^{n-1} \simeq S^{n}$. Since this upper horizontal arrow is the inverse of $\partial$, we conclude the commutativity of (5.4.3).

To prove the commutativity of (5.4.4), we consider the following diagram:

$$
\begin{array}{ccc}
\pi_{j} \mathcal{Z}_{m}\left(A \times S^{n}\right) / \mathcal{Z}_{m}(A \times\{\infty\}) & \partial \\
\pi_{j} \mathcal{Z}_{m}\left(A \wedge S^{n}\right) & \stackrel{\Sigma}{\Sigma} & \pi_{j-1} \mathcal{Z}_{m}\left(A \times S^{n-1}\right) / \mathcal{Z}_{m}(A \times\{\infty\}) \\
\mathcal{A} \downarrow & & \mathcal{A} \downarrow \\
H_{m+j}\left(\Sigma^{n} A\right) & \longleftarrow \Sigma & \mathcal{Z}_{m}\left(A \wedge S^{n-1}\right) \\
\left(\Sigma^{n}\right)^{-1} & & H_{m+j-1}\left(\Sigma^{n-1} A\right) \\
H_{m-n+j}(A) & \stackrel{\Sigma}{ } & \left(\Sigma^{n-1}\right)^{-1} \downarrow
\end{array}
$$
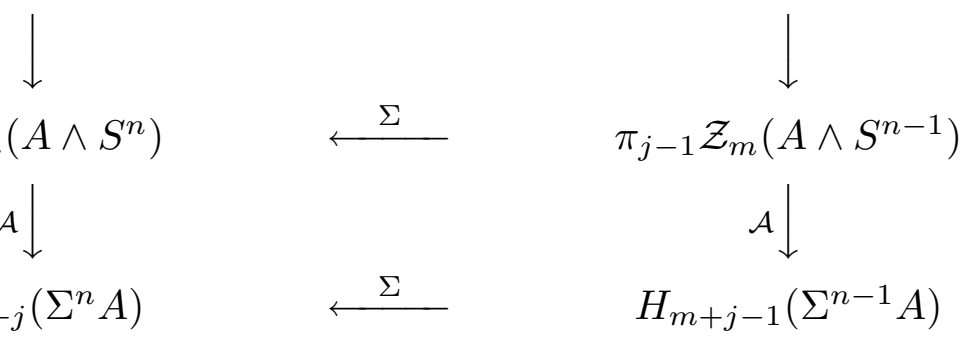

We verify that the composition of maps in the left (respectively, right) column of (5.4.5) equals $S l \circ \mathcal{A}$ by using the property that $\mathrm{Sl}$ is left inverse to $\left.(-) \times\left[S^{n}\right]\right)\left(\right.$ resp., $\left.\left.(-) \times\left[S^{n-1}\right]\right)\right)$. Thus it suffices to verify the commutativity of (5.4.5).

The upper and lower squares of (5.4.5) are seen to commute by inspection.

Recall [A;3.2] $\mathcal{A}: \pi_{i} \mathcal{Z}_{m}(X) \rightarrow H_{m+i}(X)$ is constructed by associating to a homotopy class of maps $f:\left(I^{i}, \partial I^{i}\right) \rightarrow \mathcal{Z}_{m}(X)$ a map of chain complexes $f_{*}: C_{*}\left(I^{i}, \partial I^{i}\right) \rightarrow \mathcal{I}_{*}(X)$ which raises degree by $m$, where $C_{*}\left(I^{i}, \partial I^{i}\right)$ is the cellular chain complex of a suitable subdivision of $\left(I^{i}, \partial I^{i}\right)$ and $\mathcal{I}_{*}(X)$ is the chain complex of (discrete groups of) integral currents. Then the homotopy class of $f$ is sent to $f_{*}\left(\left[I^{i}, \partial I^{i}\right]\right)$. Given $\alpha \in \pi_{j-1}\left(\mathcal{Z}_{m}(A \wedge\right.$ $\left.\left.S^{n-1}\right)\right)$ represented by $f:\left(I^{j-1}, \partial I^{j-1}\right) \rightarrow A \wedge S^{n-1}$, we realize $\Sigma \alpha \in \pi_{j}\left(\mathcal{Z}_{m}\left(A \wedge S^{n}\right)\right)$ as the homotopy class of the map $\Sigma f$ which makes the following square commute

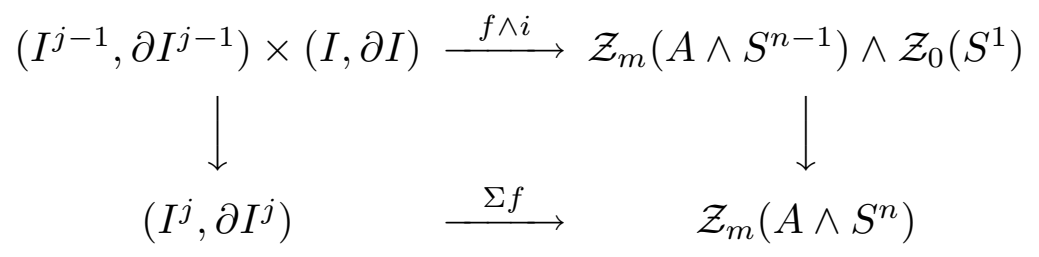


whose vertical maps are induced by taking products. This commutative diagram determines a commutative square of chain complexes (whose horizontal maps have degree $m$ )

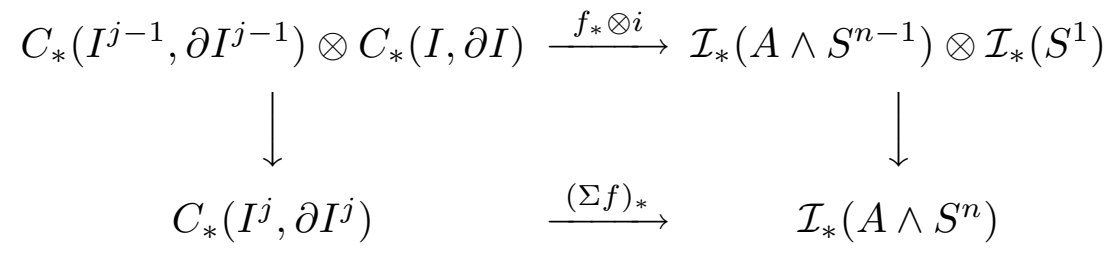

The commutativity of (5.4.6) and (5.4.7) implies the commutativity of the middle square of (5.4.5), thus completing the proof of the theorem.

\section{§6. Compatibility with the Algebro-Geometric Duality map.}

In this section we briefly discuss the duality map between algebraic cycles and algebraic cocycles established in [F-L2]. We then use the results above to prove that algebraic duality is naturally compatible with Poincaré duality with respect to the natural transformation from the algebraic theory to standard integral homology and cohomology.

Definition 6.1. [F-G], [Li1] Let $X$ be a complex projective algebraic variety. For nonnegative integers $r$ and $d$, let $C_{r, d}(X)$ denote the Chow variety of effective $r$-cycles of degree $d$ on $X$. We denote by

$$
\mathcal{C}_{r}(X) \equiv \coprod_{d \geq 0} C_{r, d}(X)
$$

the Chow monoid of effective $r$-cycles on $X$, topologized using the analytic topology of the complex projective algebraic varieties $C_{r, d}(X)$ and given the monoid structure determined by addition of cycles. We denote by

$$
Z_{r}(X) \equiv\left\{\mathcal{C}_{r}(X)\right\}^{+}
$$

the topological abelian group defined as the naïve group completion of the topological monoid $\mathcal{C}_{r}(X)$, so that the topology is that of a quotient of $\mathcal{C}_{r}(X)^{\times 2}$.

In particular, $\mathcal{C}_{0}(X)=\coprod_{d \geq 0} S P^{d}(X)$ and the underlying set of $Z_{0}(X)$ is the free abelian group on the points of $X$.

We recall that any complex projective algebraic variety $X$ admits a triangulation providing $X$ with the structure of a finite simplicial complex; moreover, this triangulation can be chosen so that a finite number of specified closed subvarieties of $X$ are subcomplexes $[\mathrm{H}]$. The resulting PL-structure is unique $[\mathrm{SY}]$.

Lemma 6.2. If $X$ is a projective algebraic variety, then there is a natural (continuous) embedding $Z_{r}(X) \subset \mathcal{Z}_{2 r}(X)$.

Proof. This follows from Lemma 1.1 and the results in $[\mathrm{H}]$ and $[\mathrm{SY}]$ mentioned above. 
Remark 6.3. As shown in $[\mathrm{F}-\mathrm{M}]$, the composition

$$
Z_{r}(X) \rightarrow \pi_{0} Z_{r}(X) \rightarrow \pi_{0} Z_{2 r}(X) \stackrel{D T}{\longrightarrow} H_{2 r}(X)
$$

is the classical cycle map sending an $r$-cycle $\zeta$ on $X$ to its cycle class $[\zeta] \in H_{2 r}(X)$, where the right-most map denotes the Dold-Thom isomorphism.

We recall the definition of algebraic cocycles introduced in [F-L1] and simplified in $[\mathrm{F}]$.

Definition 6.4. [F-L1, 3.1], [F, 1.5] Let $X$ be a complex, normal, projective algebraic variety and $Y$ a projective variety. The monoid of effective algebraic cocycles on $X$ with values in $Y$ is defined to be the topological abelian submonoid of the monoid (with the compact-open topology) of continuous maps from $X$ to some $S P^{d}(Y)$ consisting of morphisms $f: X \rightarrow S P^{d}(Y)$,

$$
\coprod_{d \geq 0} \operatorname{Mor}\left(X, S P^{d}(Y)\right) \subset \coprod_{d \geq 0} \operatorname{Map}_{\text {cont }}\left(X, S P^{d}(Y)\right) .
$$

We define

$$
\mathcal{M o r}\left(X, Z_{0}(Y)\right) \equiv\left\{\coprod_{d \geq 0} \operatorname{Mor}\left(X, S P^{d}(Y)\right)\right\}^{+}
$$

to be the naïve group completion of this topological monoid.

For any positive integer $s$, we define the topological abelian group of codimension $s$ cocycles on $X$ as the quotient

$$
\mathcal{M o r}\left(X, Z_{0}\left(\mathbb{A}^{s}\right)\right) \equiv \mathcal{M o r}\left(X, Z_{0}\left(\mathbb{P}^{s}\right)\right) / \mathcal{M o r}\left(X, Z_{0}\left(\mathbb{P}^{s-1}\right)\right)
$$

with the quotient topology.

Proposition 6.5. [F-L2] Let $X, Y$ be complex projective varieties with $X$ normal of dimension $n$. Then for any $d>0$ there is a natural algebraic continuous map

$$
\Gamma^{a l g}: \mathcal{M o r}\left(X, S P^{d}(Y)\right) \rightarrow \mathcal{C}_{n}(X \times Y) .
$$

Proposition 6.5 was established using an abstract construction which applies in situations more general than that considered above. In the following remark, we give an equivalent, but more explicit construction of $\Gamma(f)$.

Remark 6.6. $[\mathrm{F}-\mathrm{M}]$ The map $\Gamma^{\text {alg }}$ has the following concrete realization. Let $\Gamma^{0, d}(Y) \subset$ $S P^{d}(Y) \times Y$ denote the incidence correspondence consisting of pairs $(\sigma, y)$ with $y \in \sigma$. Then

$$
p: \Gamma^{0, d}(Y) \rightarrow S P^{d}(Y)
$$

is a "finite weighted map" in the sense of [F-M]: each point of $\Gamma^{0, d}(Y)$ is provided with a positive integral weighting such that the sums of weights in any fibre of $p$ equals $d$. Moreover, $S P^{d}(Y)$ has a "singular stratification" by Zariski closed subvarieties so that the 
set of weights above points of $S P^{d}(Y)$ is constant on open strata. Observe that a map from some $T$ into such an open stratum associated to the positive weights $d_{1}, \ldots, d_{k}$ determines $k$ maps $T \rightarrow Y$ with disjoint graphs, each with well defined multiplicity equal to one of the $d_{i}$ (these maps are determined by the $d$ projections $\Gamma^{0, d}(Y) \rightarrow Y$ ).

Since $X$ is irreducible, any morphism $f: X \rightarrow S P^{d}(Y)$ has the property that for some Zariski dense open subset $U \subset X$ the restriction $\left.f\right|_{U}: U \rightarrow S P^{d}(Y)$ has image in some open stratum of the singular stratification (whose Zariski closure is the smallest closed stratum containing the image of the generic point of $X$ ). Thus, if $d_{1}, \ldots, d_{k}$ are the positive integral weights associated to this stratum, then we can associate to $\left.f\right|_{U}$ the morphisms $f_{1}, \ldots, f_{k}: U \rightarrow Y$ with disjoint graphs and associated multiplicity $d_{1}, \ldots, d_{k}$. Then

$$
\Gamma^{a l g}(f)=\sum_{i=1}^{k} d_{i} \bar{\Gamma}\left(f_{i}\right)
$$

where $\bar{\Gamma}\left(f_{i}\right)$ is the Zariski closure in $X \times Y$ of the graph of $f_{i}$ in $U \times Y$.

Furthermore, if we consider some closed stratum $W \subset S P^{d}(Y)$ associated to the multiplicity $e_{1}, \ldots, e_{r}$ in the closure of the stratum with multiplicity $d_{1}, \ldots, d_{k}$ and if $Z \subset f^{-1}(W)$ is an irreducible component with open dense subset $V \subset Z$ (thereby locally closed in $X)$, then the behaviour with respect to specialization of the equidimensional cycle $\Gamma^{a l g}(f)$ above the normal variety $X$ implies that

$$
\left.\Gamma^{a l g}(f)\right|_{Z}=\sum_{j=1}^{r} e_{i} \bar{\Gamma}\left(\left.f\right|_{W, j}\right)
$$

where $f_{V}$ has associated morphisms $f_{W, 1} \ldots, f_{W, r}: V \rightarrow Y$.

Proposition 6.7. If $X$ and $Y$ are complex projective varieties, then the restriction to $\operatorname{Mor}\left(X, S P^{d}(Y)\right)$ of the graphing construction $\Gamma$ of Theorem 2.4 equals $\Gamma^{\text {alg }}$.

Proof. If $f: X \rightarrow S P^{d}(Y)$ is a morphism of complex projective varieties and if $X_{1} \subset X$ is a Zariski open subset mapping to some open stratum $V \subset S P^{d}(Y)$ of the singular stratification of $S P^{d}(Y)$ with weights $d_{1}, \ldots, d_{k}$, then the graph of $\left.f\right|_{X_{1}} ^{a n}$ as a rectifiable current as constructed in Remark 6.6 equals $\sum_{i=1}^{k} d_{i} \Gamma\left(f_{i}\right)^{a n}$. Applying the arguments given for Theorem 2.4 to the restriction of $f$ above open strata $V \subset S P^{d}(Y)$, we conclude that $\Gamma\left(f^{a n}\right)$ as defined in Theorem 2.4 equals $\left(\Gamma^{a l g}(f)\right)^{a n}$, where $\Gamma^{a l g}(f)$ is constructed in Remark 6.6.

As shown in the corollary below, $\Gamma^{t o p}$ of Theorem 3.6, when applied to an algebraic morphism of complex projective algebraic varieties, agrees with $\Gamma^{\text {alg }}$.

Corollary 6.8. With hypotheses and notation as in Theorem 3.6, let $f: A \rightarrow S P^{d}(B)$ be a Lipschitz map with the property that $\Gamma(f)$ is $\partial$-closed. Then $\Gamma^{\text {top }}(f)=\Gamma(f)$. In particular, if $f$ can be given the structure of a map of simplicial complexes or is an algebraic morphism of complex projective algebraic varieties, then $\Gamma^{\text {top }}(f)=\Gamma(f)$. 
Consequently, if $X$ is a normal complex projective variety of dimension $n$, then for each $d>0$ we have a natural commutative square

$$
\begin{array}{cc}
\mathcal{M o r}\left(X, S P^{d}\left(\mathbb{P}^{t}\right)\right)^{a n} \stackrel{\Gamma^{a l g}}{\longrightarrow} \mathcal{C}_{n}\left(X \times \mathbb{P}^{t}\right)^{a n} \\
\downarrow \\
\operatorname{Map}_{\text {Lip }}\left(X, S P^{d}\left(\mathbb{P}^{t}\right)\right) \stackrel{\Gamma^{t o p}}{\longrightarrow} \mathcal{Z}_{2 n}\left(X \times \mathbb{P}^{t}\right) .
\end{array}
$$

Proof. Let $f$ be the uniform limit of the sequence $\left\{f_{k}\right\}_{k=1}^{\infty} \subset \operatorname{Map}_{\text {Lip }}\left(A, S P^{d}(B)\right)$ of good Lipschitz maps with Lipschitz norm uniformly bounded by a constant $K$. Arguing as in the proof of Proposition 3.5 with $f, f_{k}$ replacing $f_{0}, f_{1}$, we obtain Lipschitz maps $h_{k}$ : $A \times I \rightarrow S P^{d}(B)$ such that

$$
\partial \Gamma\left(h_{k}\right)=\Gamma(f)-\Gamma\left(f_{k}\right) \quad \text { and } \quad \operatorname{Mass}\left(\Gamma\left(h_{k}\right)\right)<(1+c K)^{m}\|\| f-f_{k} \|_{\infty}
$$

where $c$ is a fixed constant. Hence,

$$
\Gamma^{t o p}(f) \stackrel{\text { def }}{=} \underset{k}{\lim _{k}} \Gamma\left(f_{k}\right)=\Gamma(f) .
$$

The commutativiy of (6.8.1) now follows immediately from the equality $\Gamma(f)=\Gamma^{t o p}(f)$ and Proposition 6.7.

As a corollary of Theorem 5.4, we conclude the following compatibility of $\mathcal{D}_{*}^{a l g}$ and cap product with the fundamental class.

Corollary 6.9. Let $X$ be a projective, normal variety of pure (complex) dimension $m$ and let $t$ be a positive integer $\leq m$. Then the following square commutes

$$
\begin{array}{ccc}
\pi_{j} Z^{t}(X)=L^{t} H^{2 t-j}(X) & \stackrel{\mathcal{D}_{*}^{a l g}}{\longrightarrow} L_{m-t} H_{2 m-2 t+j}(X)=\pi_{j} Z_{m-t}(X) \\
\Phi^{*} \downarrow & \downarrow_{*} \\
\pi_{j} \operatorname{Map}_{\text {Lip }}\left(X, \mathcal{Z}\left(S^{2 t}\right)^{o}\right)=H^{2 t-j}(X ; \mathbb{Z}) \stackrel{\cap[X]}{\longrightarrow} H_{2 m-2 t+j}(X)=\pi_{j} \mathcal{Z}_{2 m-2 t}\left(X^{a n}\right),
\end{array}
$$

where the vertcial maps are induced by the inclusions

$$
\operatorname{Mor}\left(X, Z_{0}\left(\mathbb{A}^{t}\right)\right) \rightarrow \operatorname{Map}_{\text {Lip }}\left(X, \mathcal{Z}_{0}\left(S^{t}\right)^{o}\right), \quad Z_{m-t}(X) \rightarrow \mathcal{Z}_{2 m-2 t}\left(X^{a n}\right) .
$$

If $X$ is smooth, then in particular the duality isomorphism $\mathcal{D}_{*}^{a l g}$ of [F-L2] is compatible with the classical Poincaré duality isomorphism.

Proof. We identify $Z_{0}\left(\mathbb{A}^{t}\right)$ with $Z_{0}\left(\mathbb{P}^{t}\right) / Z_{0}\left(\mathbb{P}^{t-1}\right)$ and we identify $S P^{d}\left(S^{2 t}\right)$ with $S P^{d}\left(\left(\mathbb{P}^{t}\right)^{a n} /\left(\mathbb{P}^{t-1}\right)^{a n}\right)$. It suffices to observe that Corollary 6.8 verifies the commutativity for each $d>0$ of the square (natural with respect to both $d$ and $t$ )

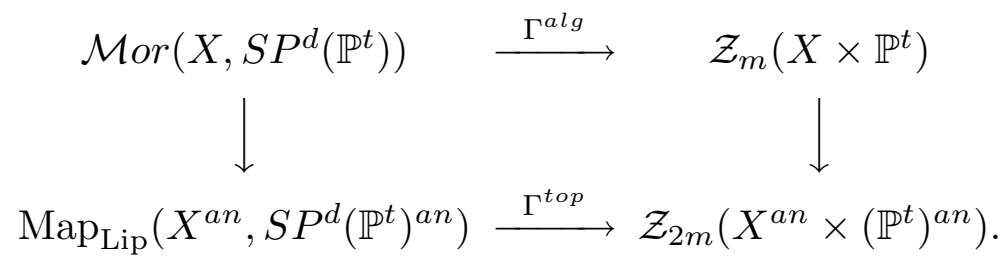




\section{References}

[A-R] R. Abraham and J. Robbin, Transversal mappings and flows, Benjamin, 1967.

[A] F. Almgren, Jr., Homotopy groups of the integral cycle groups, Topology 1 (1962), 257-299.

[D-T] A. Dold and R. Thom,, Quasifaserungen und unendliche symmetrische produkte, Ann. of Math. 67 (1956), 230-281.

[Fe] H. Federer, Geometric measure theory, Springer-Verlag, New York, 1969.

[F] E. Friedlander, Algebraic cocycles on normal, quasiprojective varieties, Compositio Math 110 (1998), 127-162.

[F-G] E. Friedlander and O. Gabber, Cycle spaces and intersection theory, Topological Methods in Modern Mathematics, 1993, pp. 325-370.

[F-L1] E. Friedlander and H.B. Lawson, Jr., A theory of algebraic cocycles, Annals of Math. 136 (1992), 361-428.

[F-L2] E. Friedlander and H.B. Lawson, Jr., Duality for spaces of algebraic cocycles and algebraic cycles, Topology 36 (1997), 535-565.

[F-M] Friedlander and B. Mazur, Filtrations on the homology of algebraic varieties, vol. 529, Memoir, A.M.S., 1994.

[F-V] E. Friedlander and V. Voevodsky, Bivariant cycle homology, in Cycles, transfers, and Motivic Homology Theories, Annals of Math Studies (2000), 138-187.

[G] P. Gajer, Poincaré duality and integral cycles, Compositio Math. 98 (1995), 193-203.

[G-M] P. Griffiths and J. Morgan, Rational homotopy theory and differential forms, Birkhauser, 1981.

[H] H. Hironaka, Triangulation of algebraic sets, Algebraic Geometry, Proc. of Symp. in Pure Math 29 (1975), 165-185.

[Hi] M. Hirsch, Differential Topology, Springer-Verlag, New York, 1976.

[H-Y] J. Hocking and G. Young, Topology, Addison Wesley, 1961.

[Ho] L. Hormander, Linear Partial Differential Operators, Springer-Verlag, New York, 1964.

[L1] H.B. Lawson, Jr., Algebraic cycles and homotopy theory, Ann. of Math. 129 (1989), 253-291.

[L2] H.B. Lawson, Jr., Spaces of algebraic cycles, in Surveys in Differential Geometry II A.M.S. (1995).

[Li1] Lima-Filho, Lawson homology for quasiprojective varieties, Compositio Math 84 (1992), 1-23.

[Li2] P. Lima-Filho, On the generalized cycle map, J. Diff. Geom. 38 (1993), 105-130.

[Mi] R. J. Milgram, The homology of symmetric products, Trans. A.M.S. 138 (1969), 251-266.

[P] H. Poincaré, Compléments à l'Analysis situs, H. Poincaré, Oeuvres, Vol VI, Gauthier-Villars, 1953, pp. 189-192.

[SY] M. Shiota and M. Yokoi, Triangulations of subanalytic sets and locally subanalytic manifolds, Trans. Amer. Math. Soc. 286 (1984), 727-750. 